Article

\title{
Diversity and life-cycle analysis of Pacific Ocean zooplankton by videomicroscopy and DNA barcoding: Crustacea
}

\author{
Peter Bryant ${ }^{1}$ and Timothy Arehart ${ }^{2}$ * \\ 1 Department of Developmental and Cell Biology, University of California, Irvine, Irvine, CA, USA; pjbryant@uci.edu \\ ORCID iD: 0000-0003-1989-3921 \\ 2 Crystal Cove Conservancy, Newport Coast, CA, USA; ponkyjoe@yahoo.com \\ * Correspondence: pjbryant@uci.edu
}

\begin{abstract}
Determining the DNA sequencing of a small element in the mitochondrial DNA (DNA barcoding) makes it possible to easily identify individuals of different larval stages of marine crustaceans without the need for laboratory rearing. It can also be used to construct taxonomic trees, although it is not yet clear to what extent this barcode-based taxonomy reflects more traditional morphological or molecular taxonomy. Collections of zooplankton were made using conventional plankton nets in Newport Bay and the Pacific Ocean near Newport Beach, California (Lat. 33.628342, Long. -117.927933) between May 2013 and January 2020, and individual crustacean specimens were documented by videomicroscopy. Adult crustaceans were collected from solid substrates in the same areas. Specimens were preserved in ethanol and sent to the Canadian Centre for DNA Barcoding at the University of Guelph, Ontario, Canada for sequencing of the COI DNA barcode. From 1042 specimens, 544 COI sequences were obtained falling into 199 Barcode Identification Numbers (BINs), of which 76 correspond to recognized species. The results show the utility of DNA barcoding for matching life-cycle stages as well as for documenting the diversity of this group of organisms.

Keywords: Crustacea; Zooplankton; Plankton; Pacific Ocean; Larvae; DNA barcoding
\end{abstract}

\section{Introduction}

Crustacea is an Arthropod taxon that includes amphipods, branchiopods, isopods, mysids, cladocerans, copepods, cumaceans, ostracods, stomatopods, tanaids, crabs, lobsters, crayfish, shrimps, prawns, krill, woodlice, and barnacles. Crustaceans are distinguished from other arthropods including insects, myriapods and spiders, mainly by the possession of biramous (apparently branched) limbs, and by their aquatic larval forms [1]. Like other arthropods, crustaceans have an exoskeleton, which is molted multiple times during growth and development. Most species are free-living and aquatic, although a few (e.g. woodlice) are terrestrial, some are parasitic, and some (e.g. barnacles) are sessile. As with other phyla in the zooplankton, Crustacea include both holoplankton (animals that remain planktonic for their entire life cycle) and meroplankton (larval stages of benthic adults). Both types are included in the present study. Illustrated guides to the zooplankton of Southern California are available [2-4]

The first larval stage in most marine crustacea is the nauplius [5], which has a simple, unsegmented body, three pairs of appendages and a single, median eye. An exception is the Isopoda, in which adults which produce fully formed young [6]After the nauplius stage, some crustaceans (Branchiopoda) [7] have direct development, with the body becoming segmented and larger but staying similar in morphology through multiple molts. Others, as in the Malacostraca [8] include some degree of metamorphosis during their development; this is most dramatic in the Decapoda [9] which go through the stages of zoea and megalops before becoming adult; and in the barnacles [10], which transform from free-swimming larvae into sessile adults. Mapping out these developmental processes has often been done by raising individual specimens in the laboratory, but the present work shows that the research can be accelerated by using DNA barcoding to match larval stages with adults.

The body of a crustacean [11] is composed of segments, which are basically grouped into three regions: head, thorax and abdomen, although the head and thorax are often fused into a cephalothorax. Each body segment can bear a pair of appendages: on the head, these include two pairs of antennae, the mandibles and maxillae. The thoracic segments bear legs, which may be specialized as walking legs or feeding appendages. 
There are about 67,000 described species of Crustacea [12]. The small marine copepods ( $<1 \mathrm{~mm}$ in length) which make up one of the subgroups of crustacea, are the most abundant metazoans on Earth [13].

Crustacea are divided into six classes [14] of which four $\left(^{*}\right)$ are included in the present study.

* Branchiopoda - consisting of four living groups, of which only one, the Cladocera ("water fleas"), will be considered here.

Remipedia - blind crustaceans living in coastal aquifers which contain saline groundwater.

Cephalocarida - horseshoe shrimp comprising only twelve benthic species.

* Maxillopoda - containing six subclasses, of which two, the Copepoda and Thecostraca (including barnacles), are considered here.

* Ostracoda - (seed shrimp) separated from other Crustacea by their bivalved carapace, undifferentiated head, and seven or less pairs of thoracic limbs. The Ostracoda are divided into five Orders, of which two, the Podocopida and Myodocopida, are extant. Some members of the Podocopida are included in the present collection.

* Malacostraca - the largest of the six classes, containing about 40,000 living species divided among 16 orders including crabs, lobsters, crayfish, shrimp, krill, woodlice, amphipods, mantis shrimp and other organisms.

The present work is part of a study of the zooplankton and benthic invertebrates of Newport Bay and the coastal waters of Southern California, using traditional taxonomy as well as molecular taxonomic methods. Crustacean specimens were collected from our local waters, and DNA barcoding [15] was used to match larval stages with adults and to document the level of biodiversity within the samples. Many of the species that also have a benthic stage in the life cycle have already been listed by the Southern California Association of Marine Invertebrate Taxonomists (SCAMIT) [16]

In a previous study [17], similar methods were used to investigate the life cycles and diversity within the hydrozoans of the same region.

DNA barcoding has been previously shown to be a successful method for identifying various crustacean taxa in the North Sea [18], southern Africa [19] and from the Estuary and Gulf of St. Lawrence [20]. A recent review reported a total number of 164 barcoding studies for crustaceans with a preference for malacostracan crustaceans, in particular Decapoda [21]

The data reported in this paper contribute to the development of a DNA barcode reference library, which will allow the rapid identification of single organisms at any developmental stage. Such reference libraries can also provide the basis for the development of more global sequencing technologies including metabarcoding and parallel sequencing of complex bulk samples including "environmental DNA", which are being developed for monitoring changes in ecosystem health. For example, in a recent study of plankton communities in the Baltic Sea, five nonindigenous species were discovered, and four of these were identified exclusively by DNA barcoding [22] 


\section{Materials and Methods}

Collections were made from 23 sites in and near Newport Beach, Laguna Beach and Dana Point, Orange County California (Figure 43), as well as one site at Avalon, Catalina Island, Los Angeles County $\left(33^{\circ} 21^{\prime} \mathrm{N} ; 118^{\circ} 19^{\prime} \mathrm{W}\right)$, one at Salton Sea Harbor $\left(33^{\circ} 25^{\prime} \mathrm{N} ; 117^{\circ} 37^{\prime} \mathrm{W}\right)$, and some from Bahia de Los Angeles, Baja California $\left(28^{\circ} 58^{\prime} \mathrm{N} ; 113^{\circ} 33^{\prime} \mathrm{W}\right)$. In the following account, only the localities outside of Orange County are identified specifically.

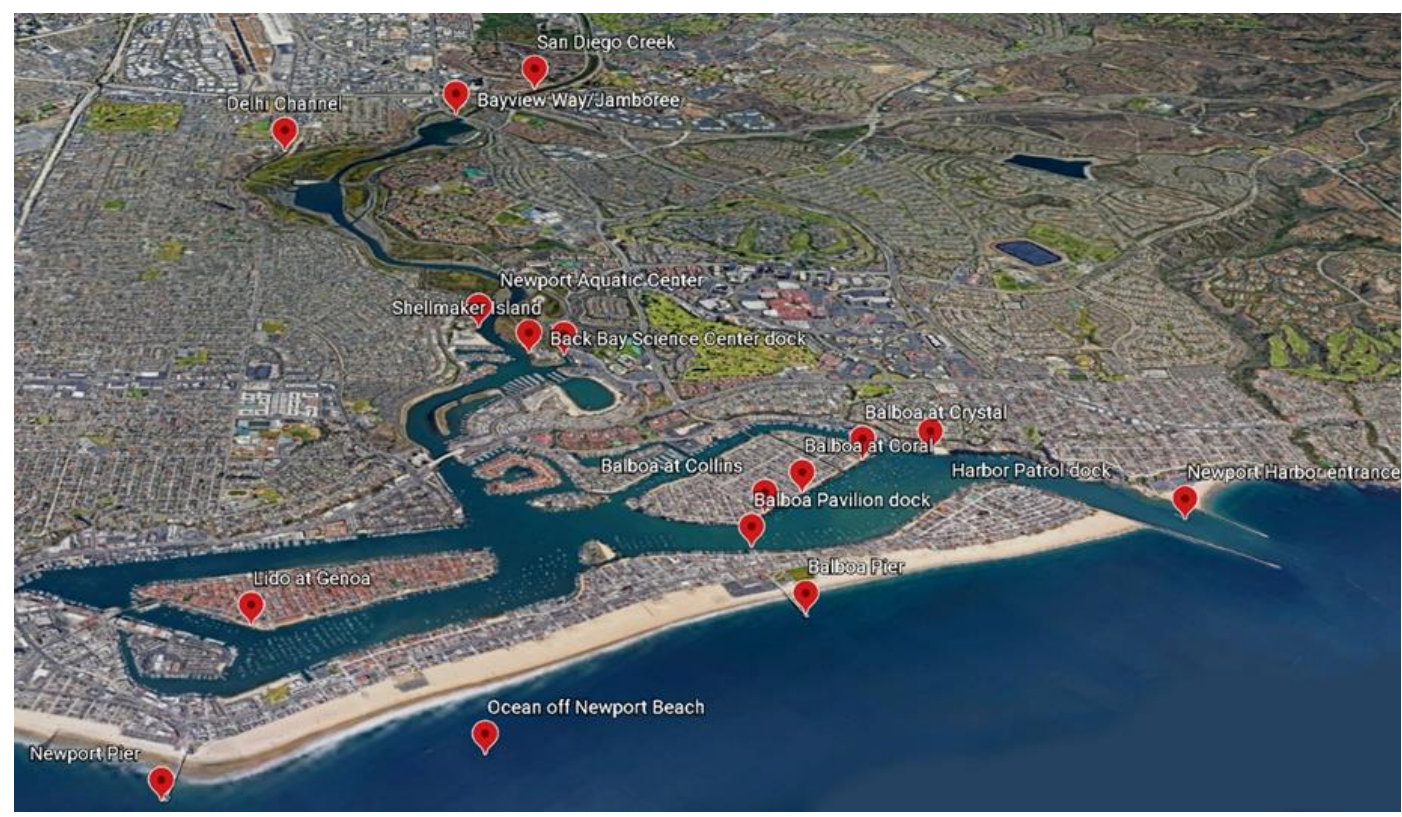

Collecting locations around Upper and Lower Newport Bay, Orange County, California

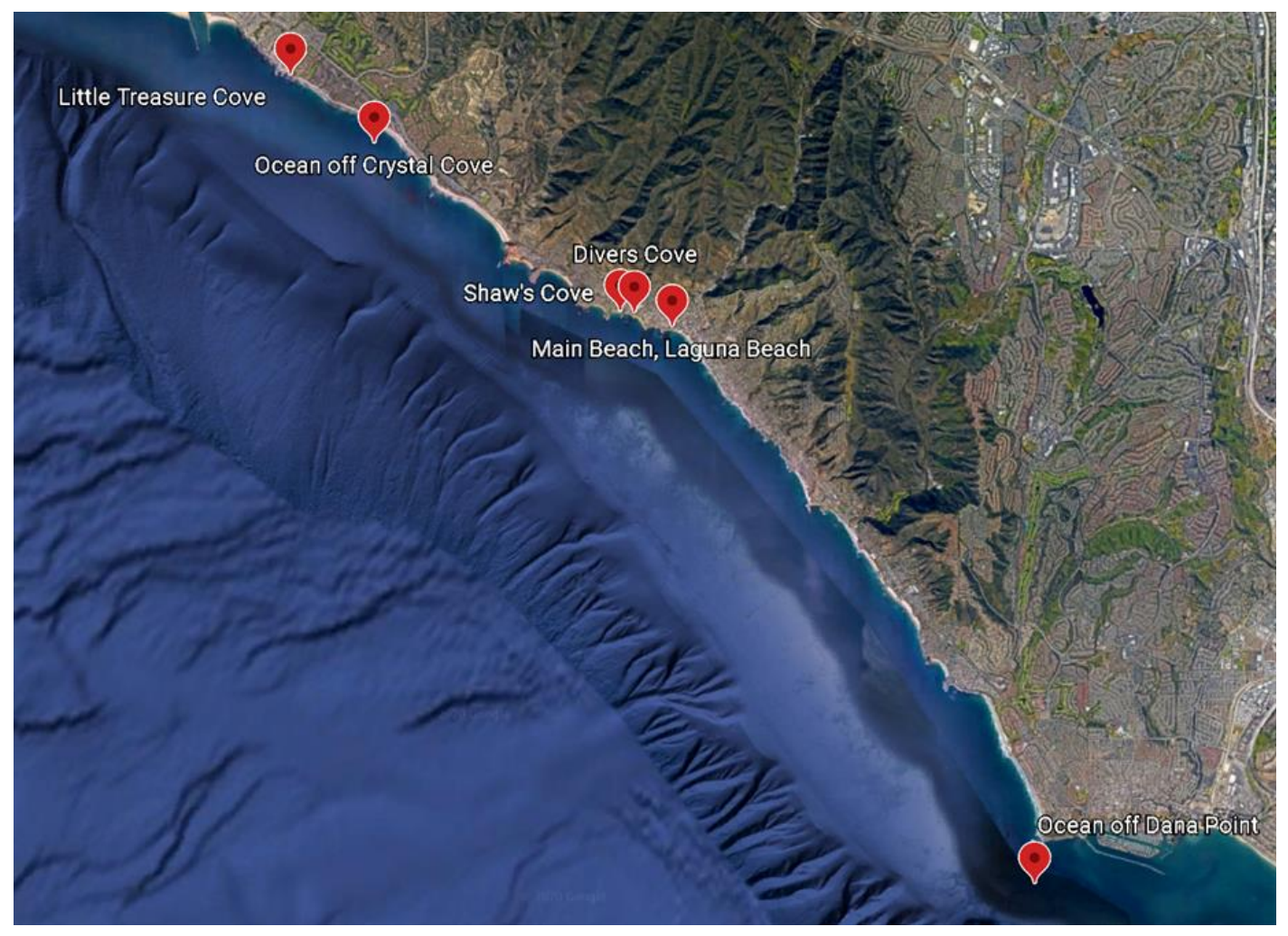

Collecting locations around Crystal Cove and Laguna Beach, Orange County, California

Shore-based collections were made with a $150 \mu \mathrm{m}$ mesh net (aperture $30 \mathrm{~cm}$ ) attached to a rope, with a $50 \mathrm{~mL}$ collection tube at the base. They were made from a public dock using repeated horizontal sweeps near the surface and 
diagonal sweeps down to about $5 \mathrm{~m}$ depth. About 5-10 sweeps of a total of about $30 \mathrm{~m}$ usually yielded sufficient specimens, but no attempt was made to monitor collections quantitatively.

Ocean collections were made with a $250 \mu \mathrm{m}$ mesh net attached to a $30 \mathrm{~m}$ rope. The net (aperture $30 \mathrm{~cm}$ ) was towed behind the vessel, just below the surface, for a period of 7 minutes at the slowest possible speed. Deployment and retrieval extended the total tow period to 10 minutes.

Plankton collections were kept in seawater, brought to the laboratory at the University of California, Irvine and examined under a dissecting microscope with lateral light and a dark background. Each specimen of interest was removed using a Pasteur Pipette, transferred to a depression slide, and recorded by video microscopy using a Zeiss microscope with a dark-field condenser, fitted with a phototube attached to a Nikon D5100 single-lens reflex camera. The most informative frames were taken from the videos and used in the figures for this paper.

Each plankton specimen was removed from the depression slide and preserved in 90\% ethanol in a single well of a 96well microplate.

Most benthic specimens (adults) were photographed at the collection site, identified, and then released. Smaller specimens were removed and brought to the laboratory to be examined and photographed under a dissecting microscope. Tissue samples were removed using dissecting tools, then transferred to the microplates for DNA sequencing.

Filled plates were sent to the Canadian Centre for DNA Barcoding at the University of Guelph, 50 Stone Road East, Guelph, ON, N1G2W1, Canada for DNA extraction using an in-house protocol (http://ccdb.ca/resources/), and bidirectional sequencing of the standard 648-bp "DNA barcode" [42] in the COI mitochondrial gene using the primers shown in Table 1. For most of the plates, we used the pair of "universal primers" which have been shown to amplify COI from a broad range of metazoan invertebrates [43]

This usually produced a DNA barcode of 658 nucleotides, and only those containing $>300$ nucleotides were included

Table 1: Primers (Degenerate bases: $S=G$ or $C ; W=A$ or $T ; R=A$ or $G$ )

\begin{tabular}{|c|c|}
\hline Forward & $\underline{\text { Reverse }}$ \\
\hline \multicolumn{2}{|l|}{ Plates BIOUG01212, CCDB-24622, CCDB-24625: } \\
\hline $\begin{array}{l}\text { ZplankF1_t1: } \\
\text { TGTAAAACGACGGCCAGTTCTASWAATCATAARGA } \\
\text { TATTGG }\end{array}$ & $\begin{array}{l}\text { ZplankR1_t1: } \\
\text { CAGGAAACAGCTATGACTTCAGGRTGRCCRAAR } \\
\text { AATCA }\end{array}$ \\
\hline \multicolumn{2}{|l|}{ Primers [43] used for all other plates: } \\
\hline LCO1490: GGTCAACAAATCATAAAGATATTGG & HCO2198: TAAACTTCAGGGTGACCAAAAAATCA \\
\hline
\end{tabular}

in the sequence analysis. They were compared with all Barcode records on BOLD with a minimum sequence length of 500bp (8,228,494 sequences) using the Bold Aligner (Amino Acid Based HMM). Groups of specimens with identical or almost identical DNA barcodes were assigned BIN numbers, where " $n=$ " after the BIN number indicates the number of DNA barcode sequences in that BIN. The identification system on BOLD delivers a species identification if the query sequence shows less than $1 \%$ divergence to a reference sequence. 
Images of all specimens as well as the DNA barcode sequences are in the public domain under code ZPC at the Canadian Centre for DNA Barcoding.

(http://www.boldsystems.org/index.php/MAS_Management_DataConsole?codes=ZPC).

\section{Results}

From 1042 specimens, 544 barcode sequences were obtained falling into 199 BINs which contained 76 recognized species (See Supplementary Data). The remaining specimens, in apparently random taxonomic groups, failed to amplify for unknown reasons.

\section{Class Branchiopoda}

Our samples (Figure 1) are all members of the order Cladocera [16]. Most cladoceran species live in fresh water, with only eight species being marine. The marine species are all in the family Podonidae, except for the genus Penilia. Our samples contained many specimens of Penilia avirostris (Family Sididae) as well as specimens of Pleopis polyphemoides, Pseudevadne tergestina, Pleopis polyphemoides, and Evadne nordmanni from the Family Podonidae [23]
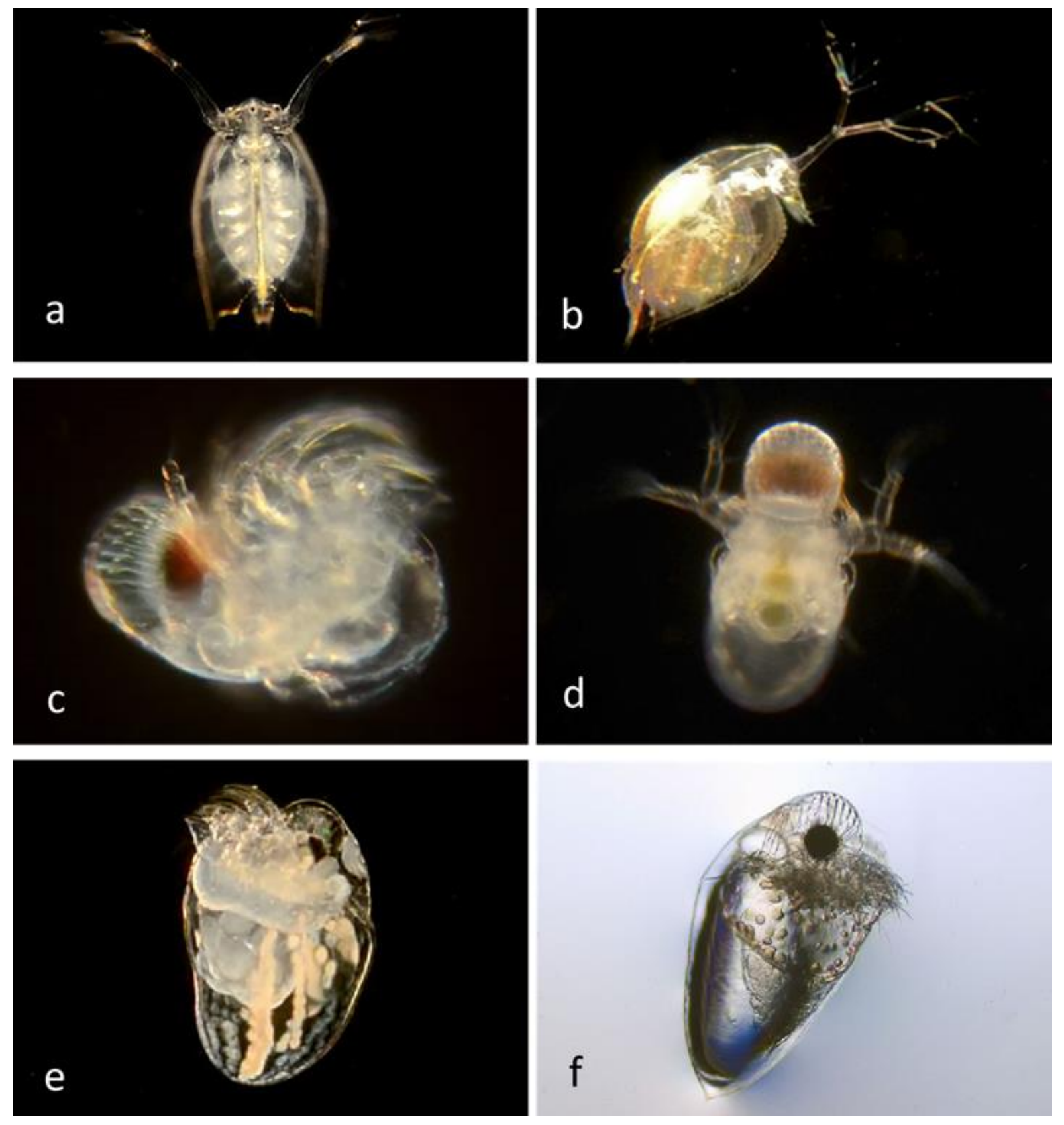
Fig. 1 Class Branchiopoda; Order Cladocera. Family Sididae: a, Penilia avirostris (BIN AAC6757; n=8) CCDB 24622 B03 off Newport Pier. b, Penilia avirostris (BIN AAC6757; n=8) BIOUG19282-C12 off Crew Dock, Upper Newport Bay. Family Podonidae: c, Pleopis polyphemoides (BIN AAD0974; n=7) BIOUG19282-H03 off Newport Aquatic Center; d, Pleopis polyphemoides (BIN AAD0974; n=7) BIOUG01212-F07 off Newport Pier; e, Pseudevadne tergestina (BIN AAE0337; n=2) CCDB 24622 C10 Newport Harbor entrance; f, Evadne nordmanni (BIN AAB7367; n=1) BIOUG01212-A03 off Crystal Cove State Park. 


\section{Class Copepoda}

Copepods are a group of small crustaceans found in nearly every freshwater and saltwater habitat on earth. Most species are holoplanktonic and they are usually the dominant members of the zooplankton, but some are benthic and some are parasitic [13]. We have found members of all three types.

The copepod egg hatches into a nauplius form, with a head and a tail but no true thorax or abdomen [24-26] (Figures $2 \mathrm{~d}, 4 \mathrm{~d}$ ). It then goes through several larval forms (Figure $4 \mathrm{~b}, \mathrm{c}$ ) separated by molts, until at the final molt it becomes adult. Development from hatching to adulthood can take from a week to a year, depending on the species and environmental conditions.

Adult females often carry sacs of eggs or embryos (Figs. 4a, 4e, 5a, 5b, 5d, 6b, 6f, 7a, 7b, 8e). In what follows, specimens are labeled as adults if they have the adult-like form, but some of those illustrated could be sub-adult stages.

Order Calanoida: Calanoid copepods are dominant in zooplankton samples from many parts of the world's oceans, making up $55 \%-95 \%$ of the total individuals [24]. The following were identified:

Family Acartiidae: four adults of Acartia tonsa (Figure 2a) and six adults of Acartia californiensis (Figure 2b).

Family Calanidae: four adults (Figure 2c) and one nauplius (Figure 2d) of 2.
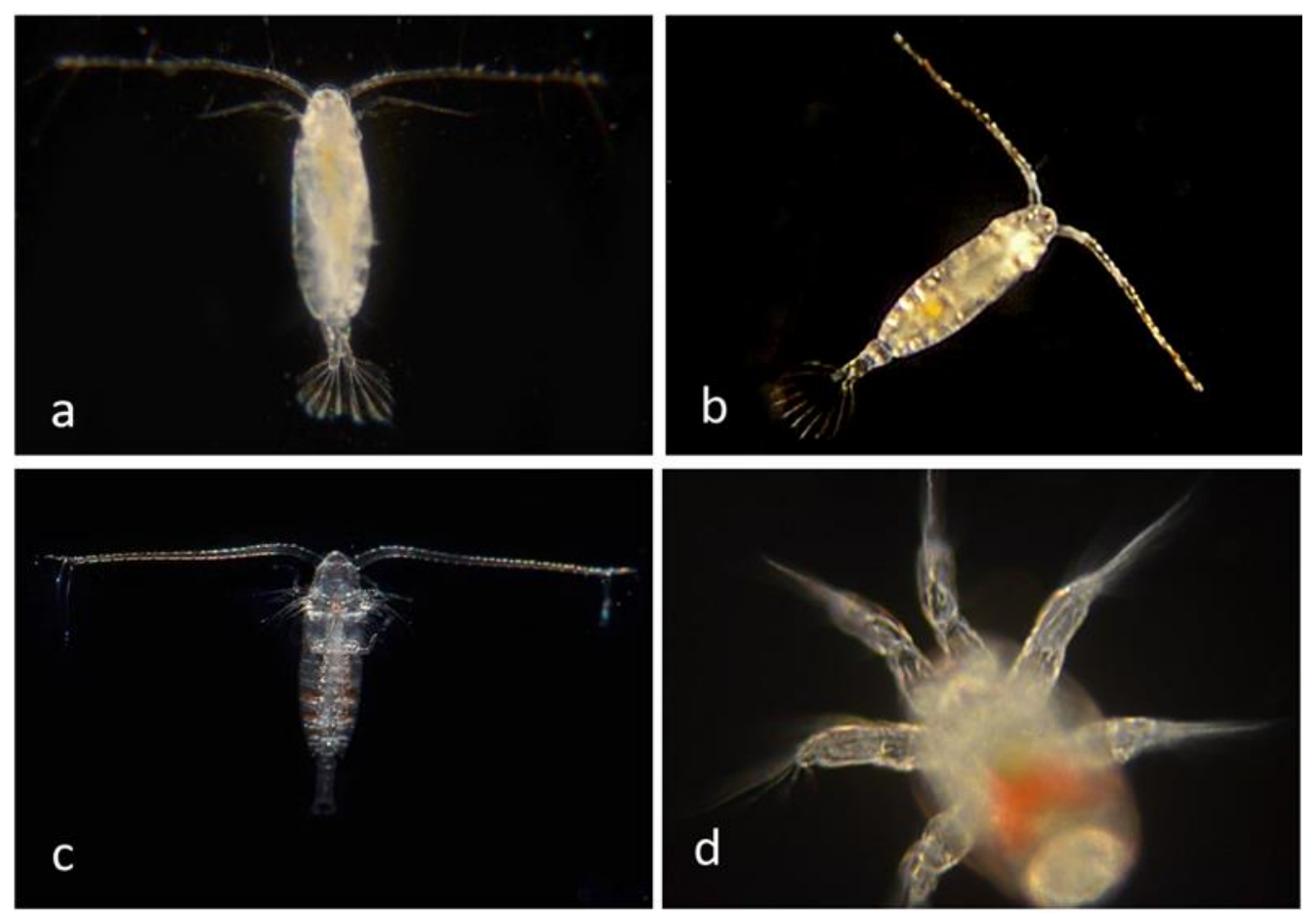

Fig. 2 Class Copepoda; Order Calanoida; Family Acartiidae: a, Acartia tonsa (BIN ACG7661, n=4). Adult

BIOUG01212-F11 from Newport Pier. b, Acartia californiensis (BIN ACH6087, n=6). Adult BIOUG01218 B02 from Back Bay Science Center dock. Family Calanidae: c, Calanus pacificus (BIN AAE8453; n=4 adults and one nauplius). Adult BIOUG01212-B10 off Avalon, Catalina Island. d, Calanus pacificus (BIN AAE8453) Nauplius BIOUG19282-D12 Ocean from Crystal Cove Alliance.

Family Paracalanidae: two adults of Mecynocera clausi with spectacularly long antennae (Figure 3a); six adults of Paracalanus parvus (Figure 3b); three adults of Paracalanus indicus (Figure 3c, 3d) all from Bahia de Los Angeles, Baja California; one adult of Parvocalanus crassirostris (Figure 3e) and one unidentified family member adult (Figure 3f). 

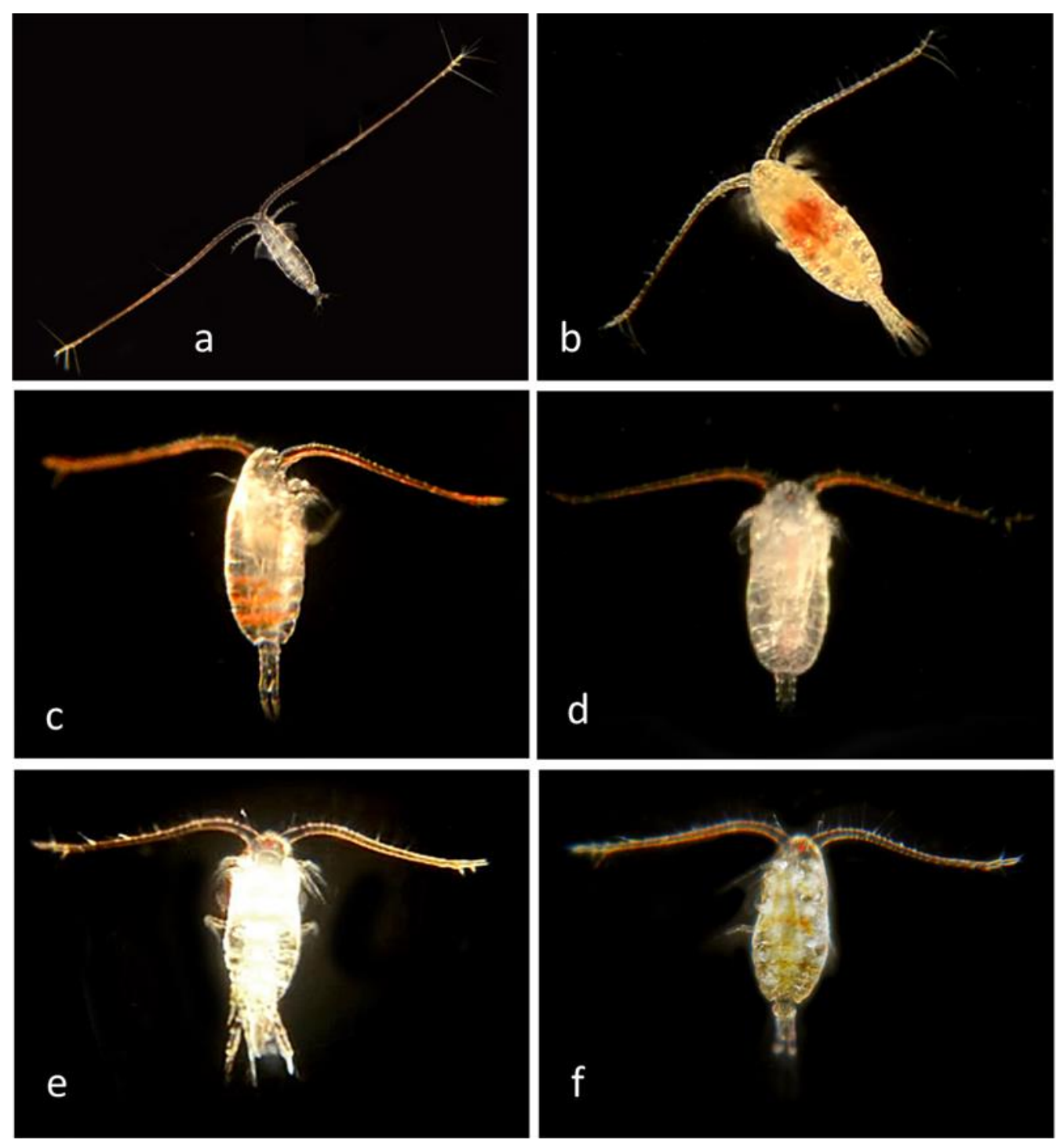

Fig. 3 Family Paracalanidae: a, Mecynocera clausi (BIN AAK7057; n=2). Adult BIOUG19282-A08 off Newport Pier. b, Paracalanus parvus (BIN ACK9448; n=5). Adult BIOUG01212-B11 Off Avalon, Catalina Island. c, Paracalanus indicus (BIN ADM8069; n=1). Adult CCDB 24625 E07. Bahia de Los Angeles, Baja California. d, Paracalanus indicus (BIN AEB8869; n=2). Adult CCDB 24625 E08. Bahia de Los Angeles, Baja California. e, Parvocalanus crassirostris (BIN ACI0538; n=1). Adult BIOUG19282-C11. Crew Dock, Upper Newport Bay. f, Paracalanidae (BIN ACY8206; n=1). Adult BIOUG19282-B08. Crew Dock, Upper Newport Bay.

Family Pontellidae: three adults (Figure 4a) and two larvae (Figure 4b) of Labidocera johnsoni as well as a larva and two adults of an unidentified family member (Figure 4c; larva; Figure $4 \mathrm{~d}$ and $4 \mathrm{e}$, adults); and one male adult of another unidentified family member; the latter showing very clearly how males of this species have one antenna significantly swollen (Figure $4 \mathrm{f}$ ). 

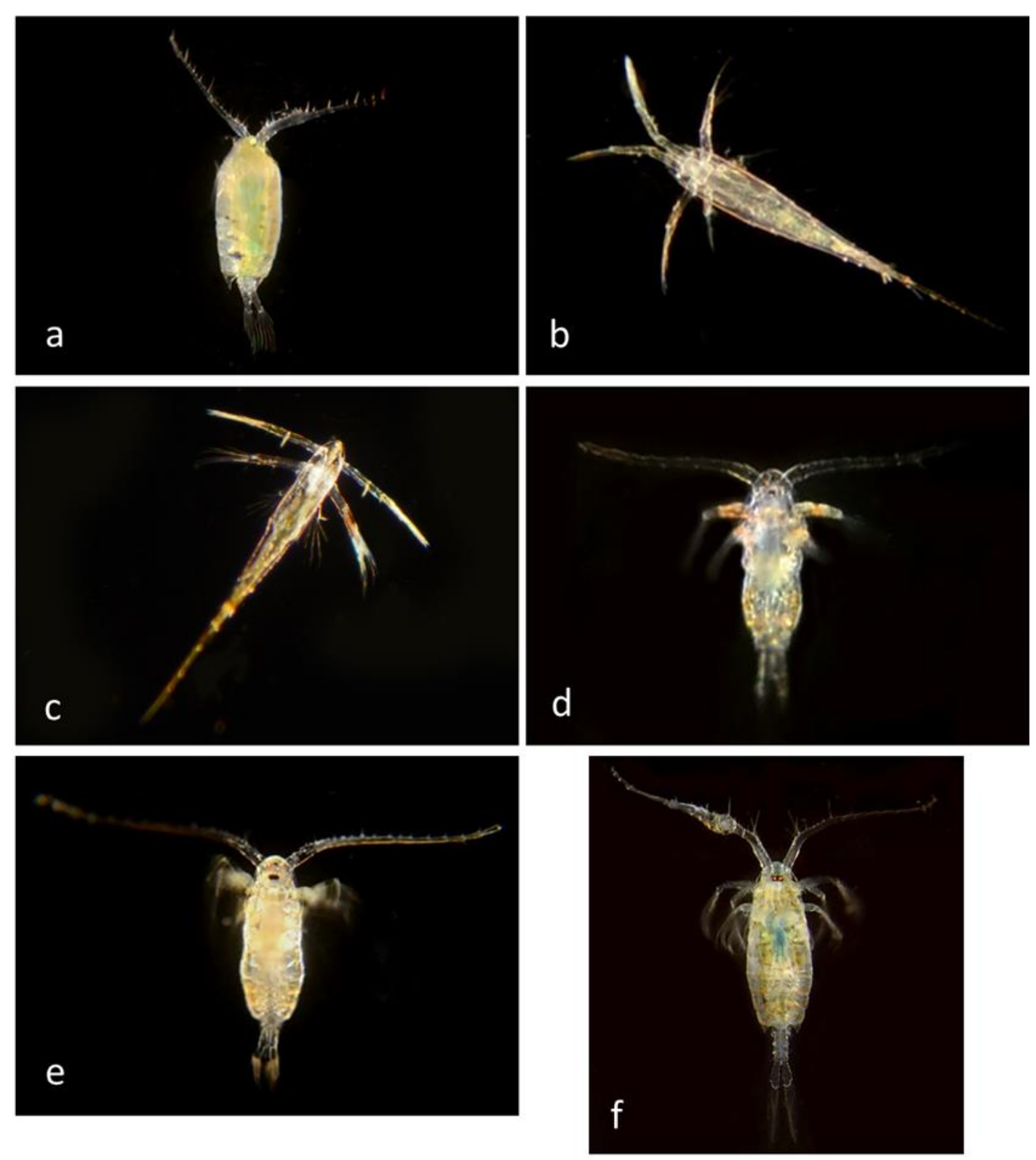

Fig. 4 Family Pontellidae, a, Labidocera johnsoni (BIN AAN5718; n=3 adults and 2 larvae). Adult BIOUG19282-G08. Pacific Ocean off Dana Point. b, Labidocera johnsoni (BIN AAN5718; n=3 adults and 2 larvae). Late nauplius larva BIOUG19282-G06 Pacific Ocean off Dana Point. c, Pontellidae (BIN ACM3770; n=1 nauplius and 2 adults). Late nauplius larva BIOUG01212-H05. Pacific Ocean off Dana Point. d, Pontellidae (BIN ACM3770; n=1 nauplius and 2 adults). Adult CCDB 24622 B01, from Balboa at Coral. e, Pontellidae (BIN ACM3770; n=1 nauplius and 2 adults). Adult. BIOUG19282-A11, from Pacific Ocean off Dana Point. f, Pontellidae (BIN ACU1959; n=2). Adult male BIOUG01226-A10, from Pacific Ocean off Dana Point.

Family Pseudodiaptomidae: one adult female with eggs of Pseudodiaptomus marinus (Figure 5a).

Family Temoridae: one adult (Figure 5b) of Temora discaudata.

Family Tortanidae: one adult (Figure 5c) and one nauplius larva (Figure 5d) of Tortanus discaudatus.

Unidentified Calanoids: five specimens of one putative species including a female with two egg cases (Figure 5e) and a mating pair (Figure 5f) showing the enlarged antenna of the male (the smaller of the two individuals). 

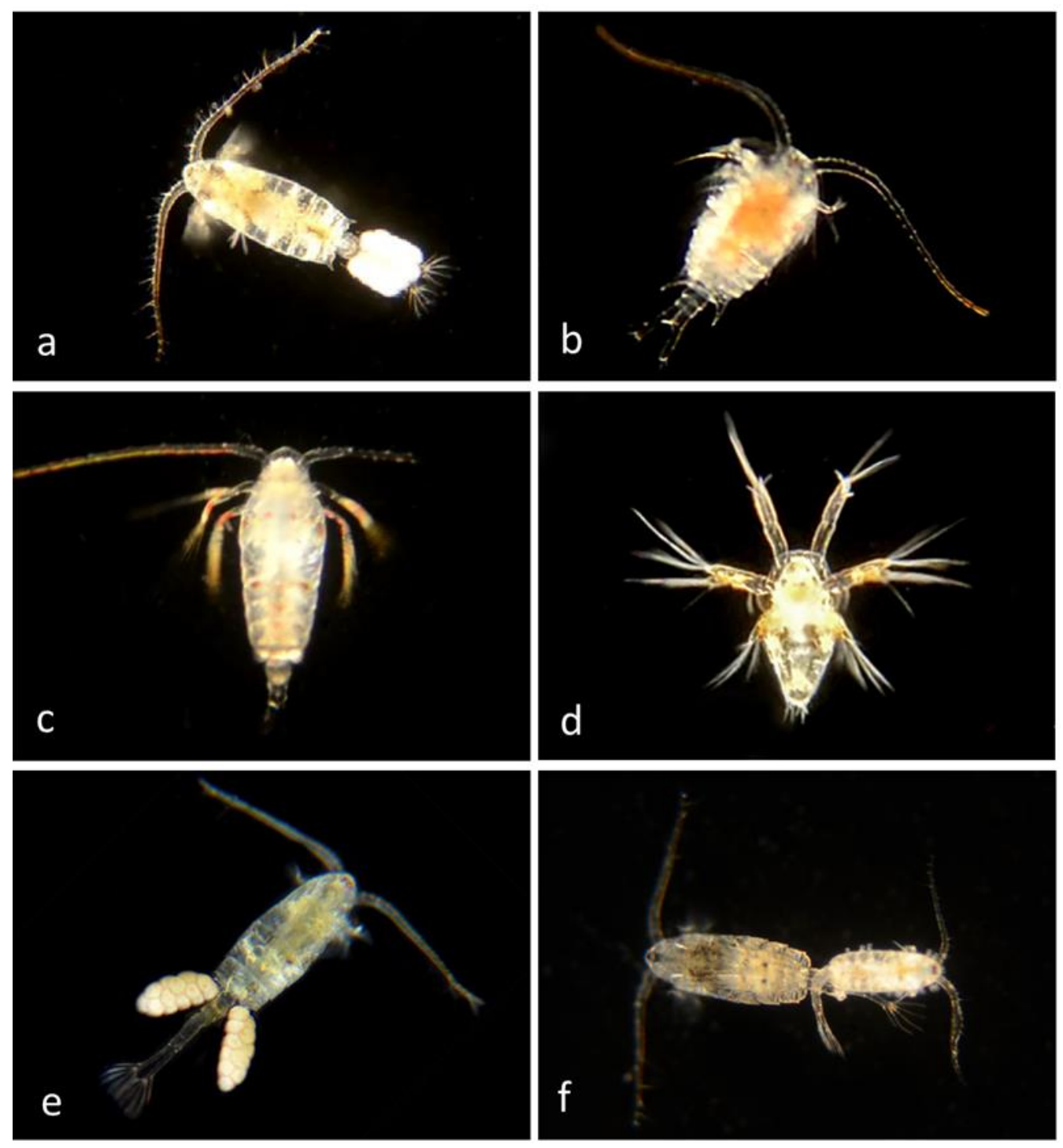

Fig. 5 a, Family Pseudodiaptomidae: Pseudodiaptomus marinus (BIN AAX6988; n=1). Adult female with eggs BIOUG01226-D10 Off Newport Pier. b, Family Temoridae: Temora discaudata (BIN AAE9776; n=1). Adult BIOUG01226-D09 From Avalon harbor, Catalina Island. c,d: Family Tortanidae: c, Tortanus discaudatus (BIN ACL8332; n=1 adult and 1 nauplius) adult CCDB 24622 D12 From Newport Harbor entrance. d, Tortanus discaudatus (BIN ACL8332; n=1 adult and 1 nauplius) Nauplius BIOUG01218 B03 from Back Bay Science Center Dock. e,f: Other Calanoida (unidentified): e, Calanoida (BIN ACM 3768; n=4). Adult female with eggs BIOUG01226-F01 from Delhi Channel, Upper Newport Bay. f, Calanoida (BIN ACM 3768; n=4) Mating pair BIOUG01212-A08 from Delhi Channel, Upper Newport Bay.

\section{Order Cyclopoida:}

Family Corycaeidae: three adults of Ditrichocorycaeus anglicus which, like other members of the family, have two large anterior eyes. Figure 6a, female carrying eggs.

Family Oithonidae: two female adults, both carrying eggs, of Oithona davisae. In the individual shown here (Figure $6 b)$, developing embryos with red eye spots can be seen inside the egg shells.

Unidentified cyclopoids: Figure 6c: Unidentified cyclopoid adult. Figure 6d: Unidentified cyclopoid, adult female with eggs. Figure 6e: Tiny copepod on seaweed. 
Family Clausidiidae: Figure 6f: Clausidium californiense adult.
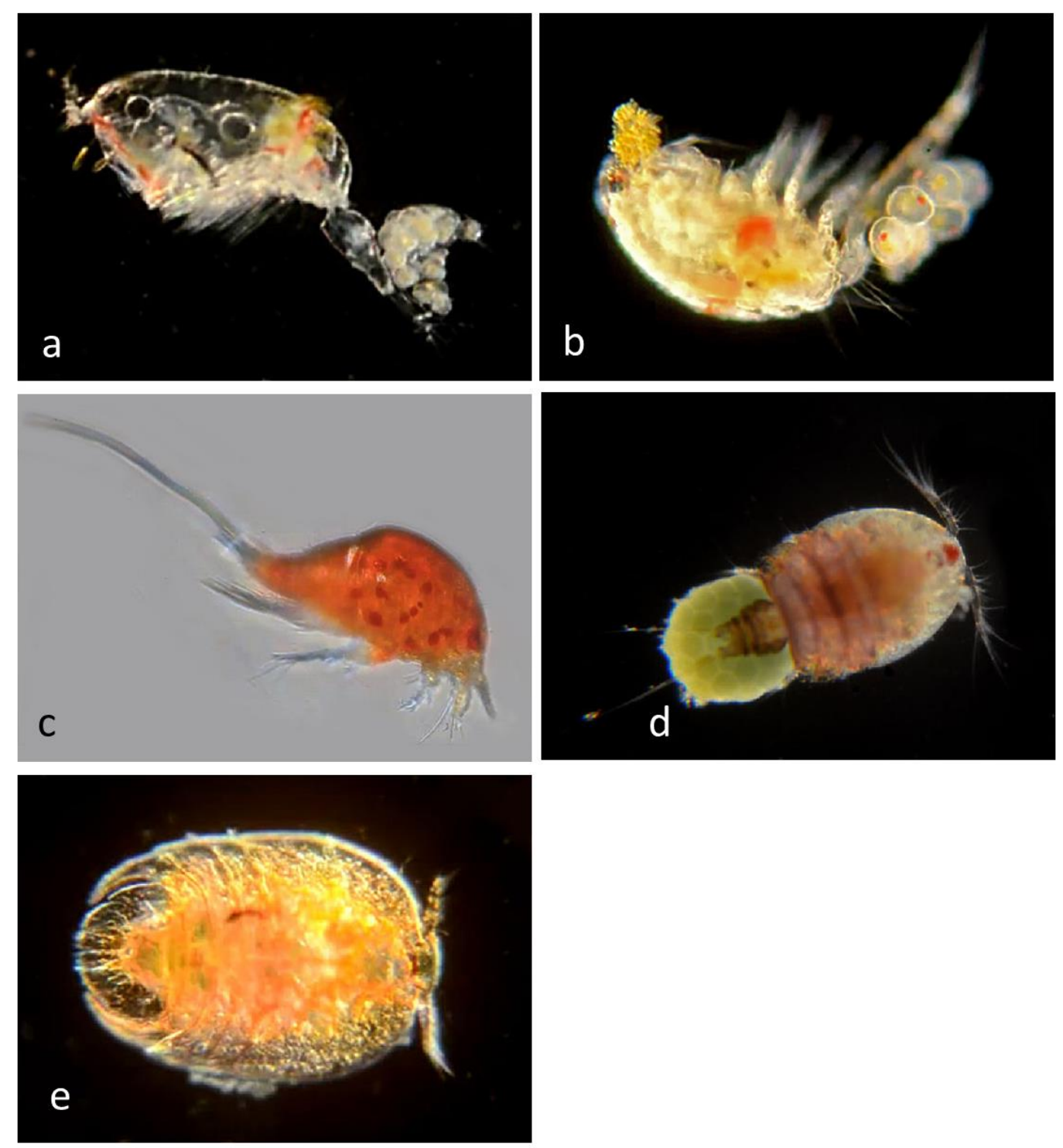

Fig. 6 Order Cyclopoida; a, Family Corycaeidae. Ditrichocorycaeus anglicus (BIN ACM3619; n=2). Adult female with eggs CCDB 24622 D07 from Newport Harbor entrance. b, Family Oithonidae: Oithona davisae (BIN ACK8948; n=2) Adult with eggs BIOUG01218 B04 off Back Bay Science Center dock. c,d,e: Unidentified cyclopoids. c, Unidentified Cyclopoid (No BIN) Adult BIOUG01212-D10 off Newport Aquatic Center. d, Unidentified Cyclopoid (BIN ACK9144; $\mathrm{n}=4$ ). Adult female with eggs BIOUG19282-E06 off Lido at Genoa. e, Tiny copepod on seaweed (BIN ADM 7440; $\mathrm{n}=2$ ) CCDB $24625 \mathrm{H} 11$ from Balboa at Coral.

Family Cyclopidae provided many examples of unidentified species, as follows: Figure 7a, Adult. Figure 7b, unidentified female carrying embryos. Figure 7c, Adult. Figure 7d, Adult. Figure 7e, Adult. Figure 7f, Adult female with eggs. 

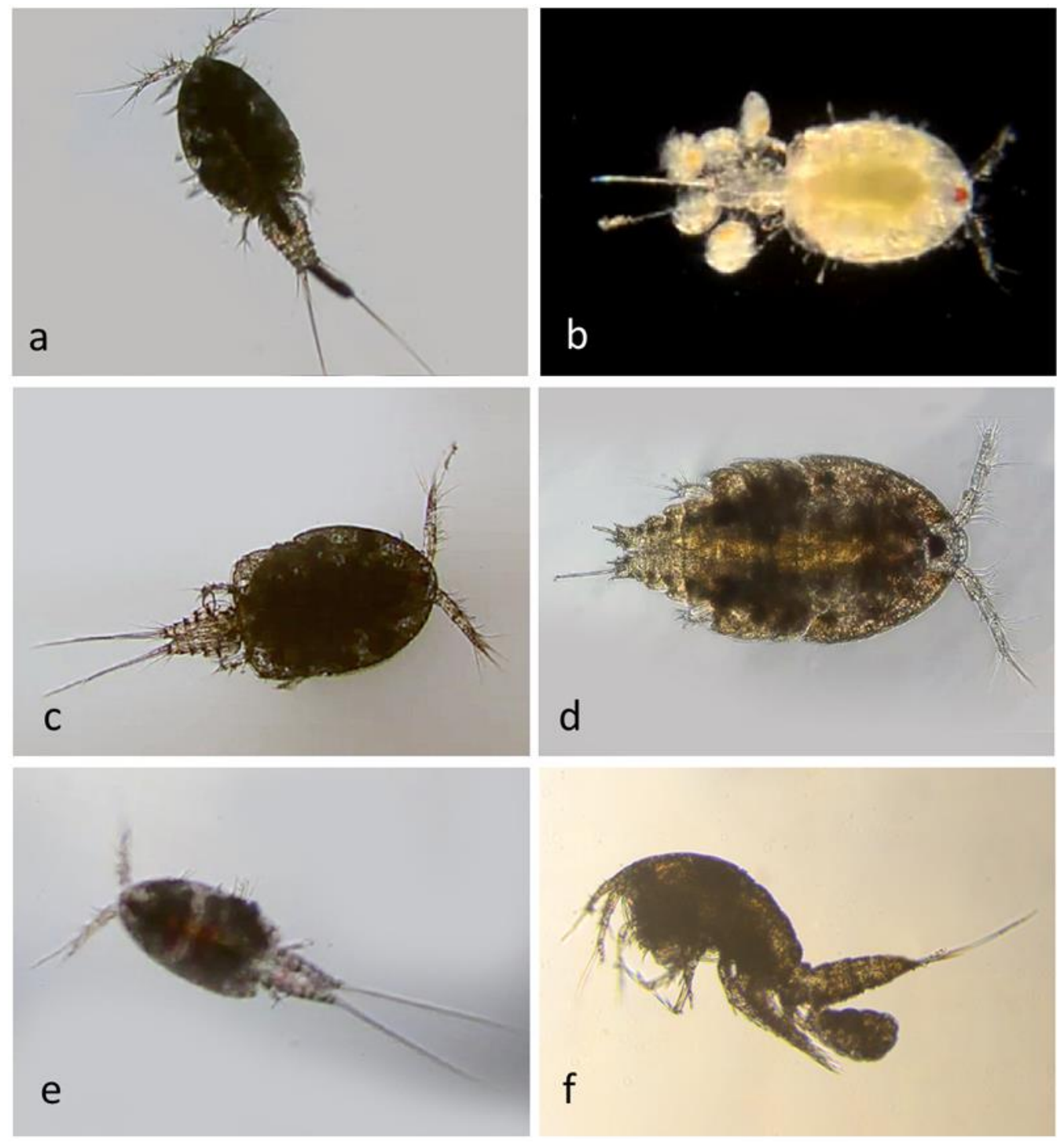

Fig. 7 Family Cyclopidae. a, Unidentified Cyclopoid (no BIN) Adult BIOUG01212-C09. Off Newport Aquatic Center, Upper Newport Bay. b, Unidentified Cyclopoid (BIN ADV1497; n=1). Female carrying embryos CCDB 24622 E09. From Shaw's Cove, Laguna Beach. c, Unidentified Cyclopoid (BIN ACM3629; n=1). Adult BIOUG01212-C02. From Divers Cove, Laguna Beach. d, Unidentified Cyclopoid (BIN ACM 3224; n=1). Adult BIOUG01212-C03. From Divers Cove, Laguna Beach. e, Unidentified cyclopoid (BIN ACK9554; n=1) Adult BIOUG01207-C11. From Balboa at Coral. f, Unidentified cyclopoid (BIN ACN1320; n=1). Female with eggs BIOUG01218 C05. From Balboa at Coral.

Order Harpacticoida. Most of these copepods are benthic, but some are planktonic so show up in our net samples.

Family Ectinosomatidae. Microsetella rosea, female with eggs (Figure 8a).

Family Thalestridae. Eudactylopus spectabilis, female with eggs (Figure 8b). Eudactylopus spectabilis, adult (Figure 8c). There are two other BINs identified as Eudactylopus spectabilis (Figure 8d) ACN1431 (n=2) and ACY 7593, (n=1).

Family Cletodidae. Cletocamptus deitersi, adult from Salton Sea harbor (Figure 8e).

Family unknown. Unidentified adult (Figure 8f). 

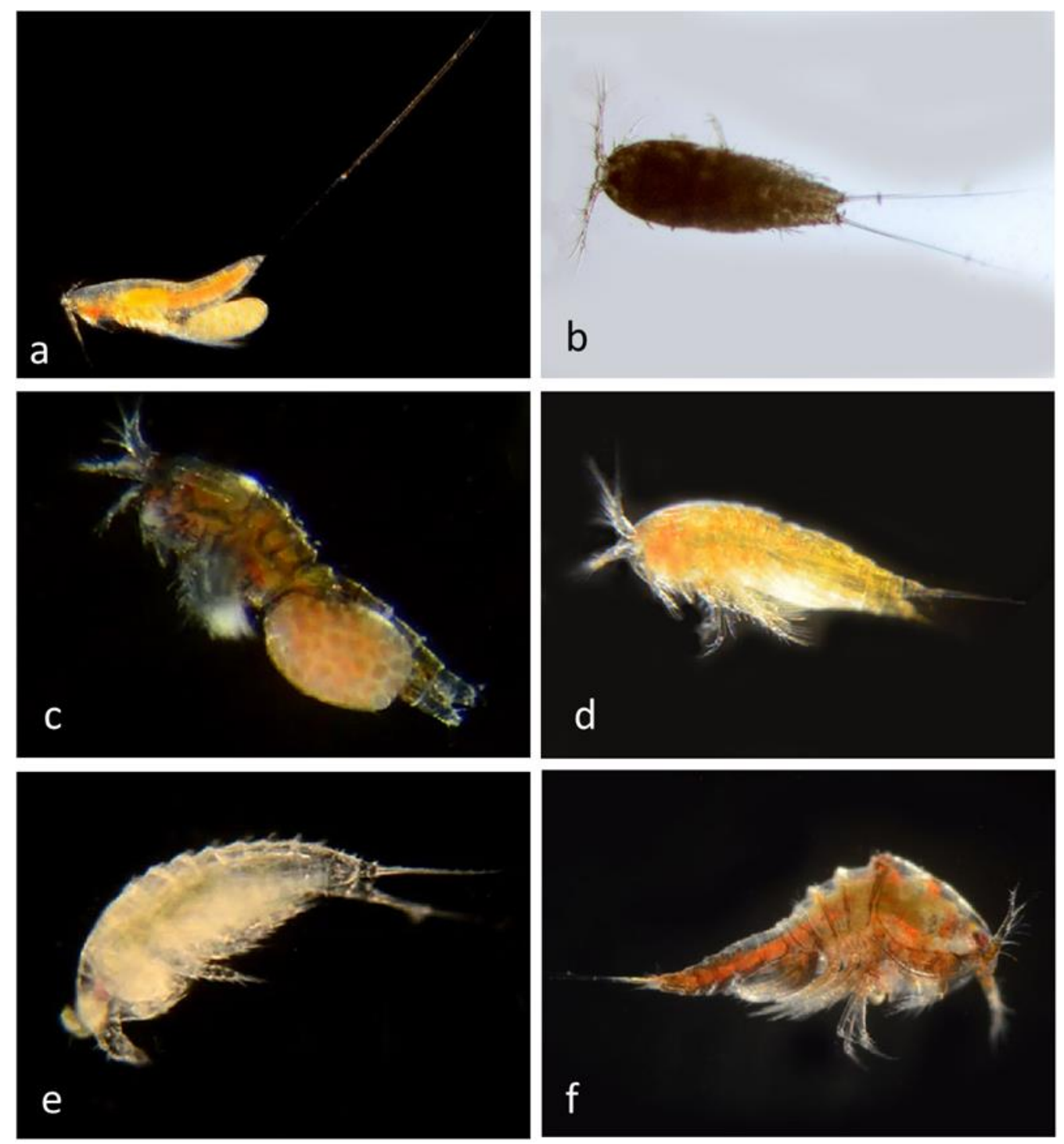

Fig. 8 Order Harpacticoida. a, Family Ectinosomatidae. Microsetella rosea female with eggs. CCDB-32335 C12 from Newport Harbor entrance. b. Family Harpacticidae. Harpacticella sp. (97\% match to Harpacticella jejuensis). c, Family Thalestridae. Eudactylopus spectabilis (BIN ACY8408; n=2). Female with eggs BIOUG01218 B11 from Lido at Genoa. d, Eudactylopus spectabilis (BIN ACY8408; n=2). Adult BIOUG19282-F07 from Crew Dock, Upper Newport Bay. e, Family Cletodidae. Cletocamptus deitersi (BIN AAE2495; n=1). Adult BIOUG19282-C02 from Salton Sea harbor. f, Family unknown. Unidentified (BIN ADV3892; n=1). Adult CCDB 24622 G04 from Newport Harbor entrance.

Family unknown. Unidentified adult (Figure 9a). Unidentified adult (Figure 9b). Unidentified adult (Figure 9c) appears to have two large anterior lenses, like Ditrichocorycaeus anglicus and other members of the Family Corycaeidae (see Figure 6a). Unidentified adult (Figure 9d). Unidentified female with eggs (Figure 9e). Unidentified adult (Figure 9f). 

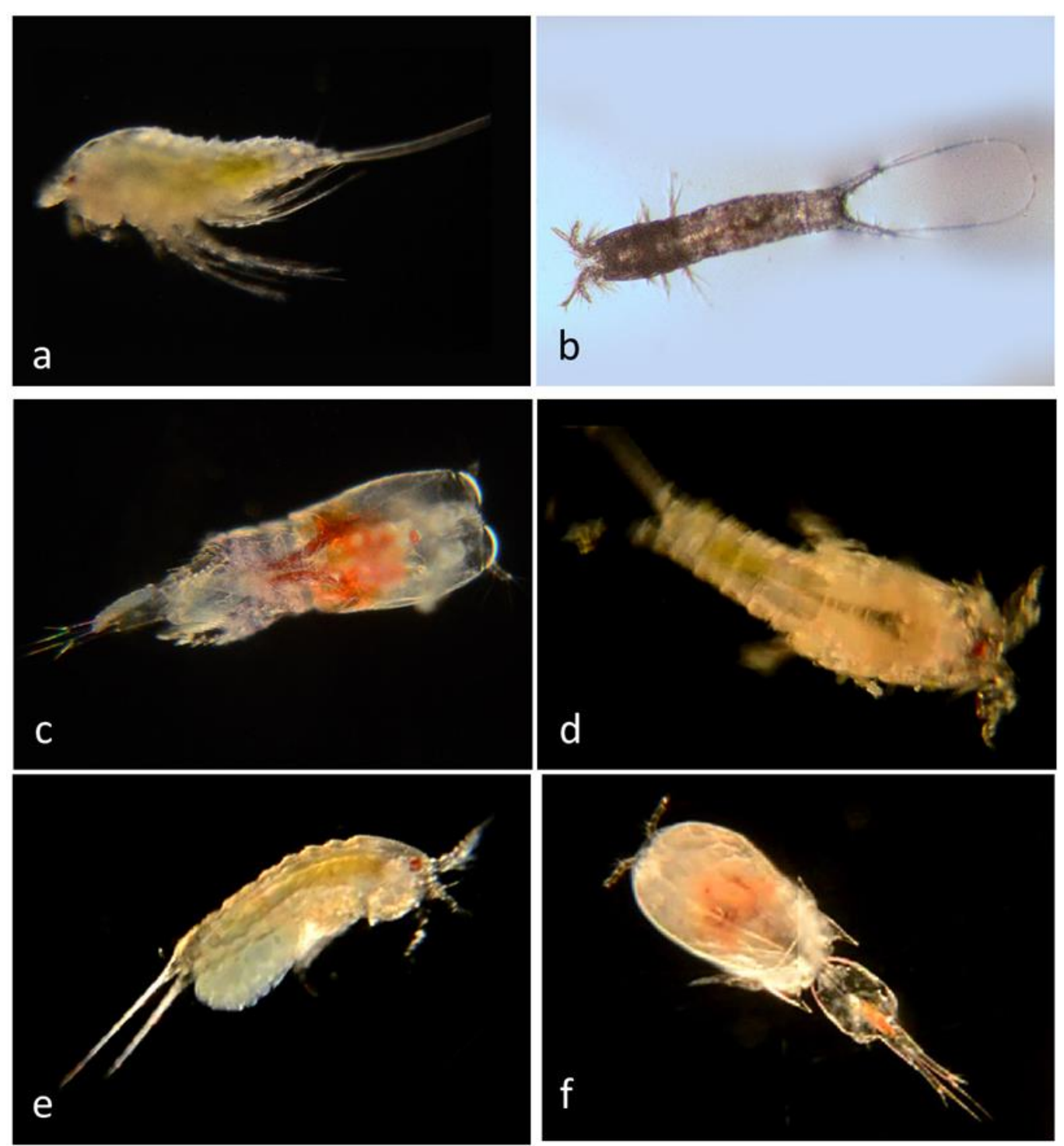

Fig. 9 Order Harpacticoida, Family unknown. a, Unidentified (BIN ACM3540; n=1). Adult BIOUG01212-H04 from Delhi channel, Upper Newport Bay. b, Unidentified (BIN ACM3541; n=1). Adult BIOUG01212-H01 off Newport Aquatic Center. c, Unidentified (BIN ACM3838; n=1) from Lido at Genoa. Appears to have two large anterior lenses, like Ditrichocorycaeus anglicus and other members of the Family Corycaeidae (see Figure 6a). Adult BIOUG01212-E02 from Lido at Genoa. d, Unidentified (BIN ACN1432; n=2). Adult BIOUG01218 A11 from Lido at Genoa. e, Unidentified (BIN ACN1432; n=2). Female with eggs BIOUG01218 G01 from Lido at Genoa. f, Unidentified (BIN ADM7045; n=1). Adult CCDB 24625 E11 at Bahia de Los Angeles, Baja California.

Order Siphonostomatoida. This order contains about 75\% of the copepods that parasitize fishes. They possess siphon-like mandibles and a frontal filament that allows attachment to their hosts. Family Caligidae. Caligus olsoni, parasitic on fish (Figure 10a).

Another group of crustaceans that are ectoparasites of fish belong to the subclass Branchiura (fish lice; Figure 10b). They are usually interpreted as members of the class Maxillopodia, which also includes Copepods but is not included in the taxonomy used in this paper. 

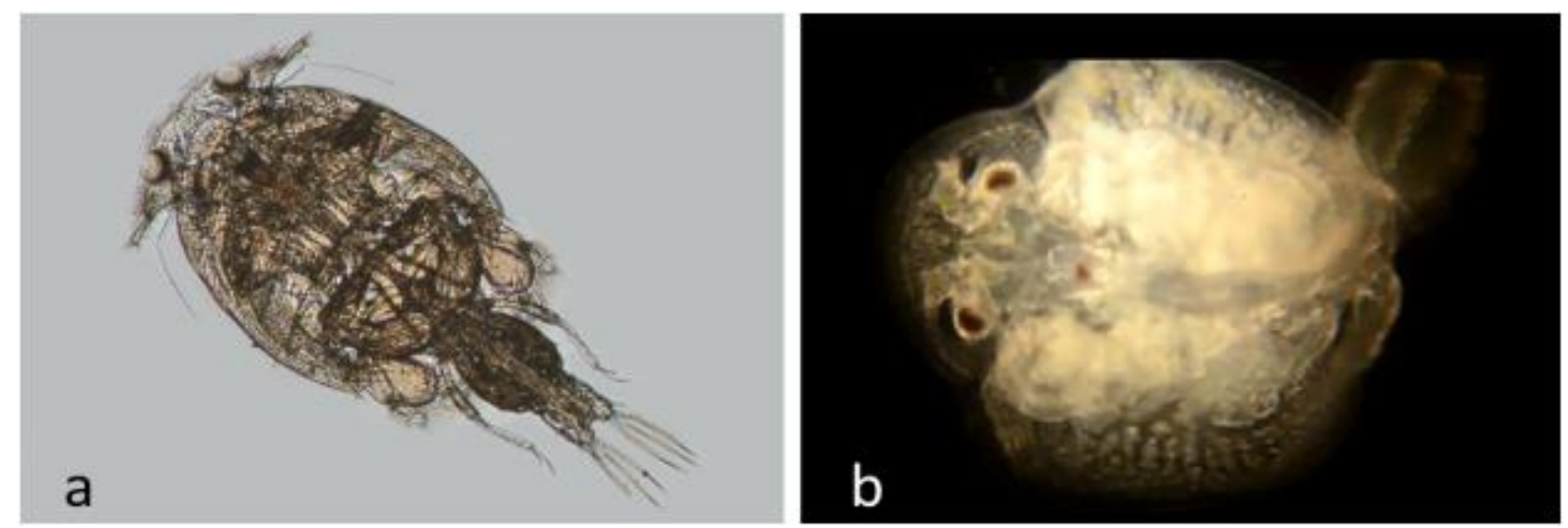

Figure 10 a, Order Siphonostomatoida. Family Caligidae. Caligus olsoni (BIN ACN1884; n=2). BIOUG01218 E05. Off Newport Aquatic Center, Upper Newport Bay. Parasitic on fish. b, Branchiura (BIN ACY8682; n=1) BIOUG19282-D03 (Dorsal). From Pacific Ocean off Newport Beach. Parasitic on fish.

\section{Class Thecostraca}

Order Sessilia Acorn barnacles [27].

Family Balanidae. Ivory barnacle, Amphibalanus eburneus [16]nauplius larva (Figure 11a). Native to the Western Atlantic, invasive to many other places. First population discovered in the US in 2000 [28-30] in the Colorado Lagoon, Long Beach, California . Amphibalanus amphitrite [16] nauplius larva (Figure 11b); Megabalanus rosa, cypris larva (Figure 11c); Megabalanus rosa, adult from Los Angeles County Museum of Natural History (Figure 11d); Paraconcavus pacificus [16], adults found on the back of a crab brought in by fishermen (Figures 11e,f) 

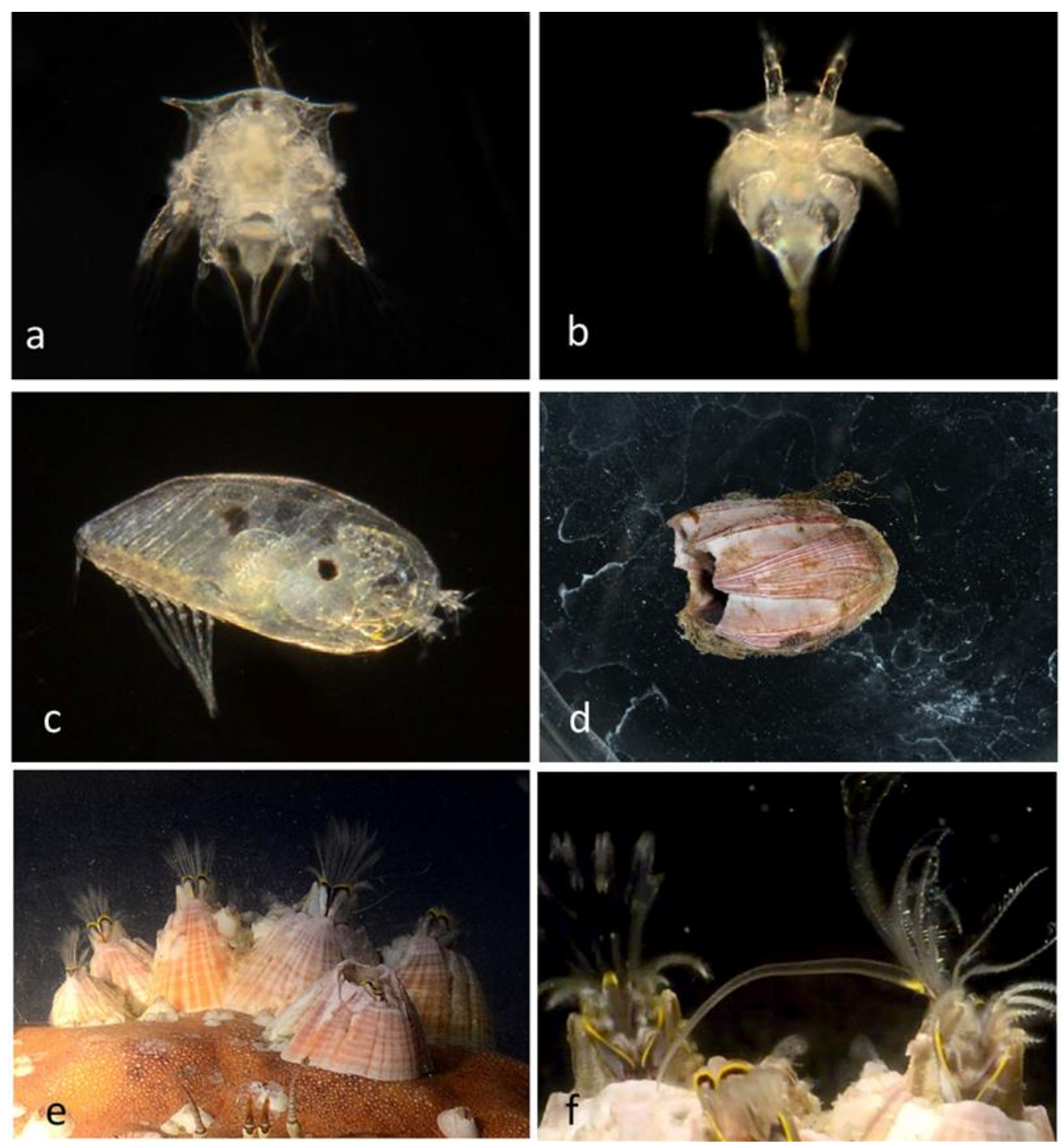

Fig. 11 Class Maxillopoda; Subclass Thecostraca; Infraclass Cirripedia; Order Sessilia; Family Balanidae: a, Amphibalanus eburneus (BIN ACD3190; n=4) BIOUG01212-A07 nauplius larva from Delhi Channel, Upper Newport Bay; b, Amphibalanus amphitrite (BIN AAO4493; n=1 nauplius larva from Lido Island at Genoa, 2 adults from the Salton Sea) BIOUG01226-C07 nauplius. c, Megabalanus rosa, cypris larva CCDB-31777 G12 at Newport Harbor entrance. d, Megabalanus rosa, adult (BIN ADK0326 from Los Angeles County Museum of Natural History). e,f, Paraconcavus pacificus, (No DNA sequence) living on a red crab brought in by fishermen at Newport Pier, Newport Beach, Orange County, CA. 10-26-2013. e, Adults feeding; f, Adults mating. 


\section{Class Ostracoda $[16,31]$}

All but two of our samples for which DNA sequences were obtained (Figure 12) are members of the order Podocopida $[16,31]$. Two species in this family have been listed [16].
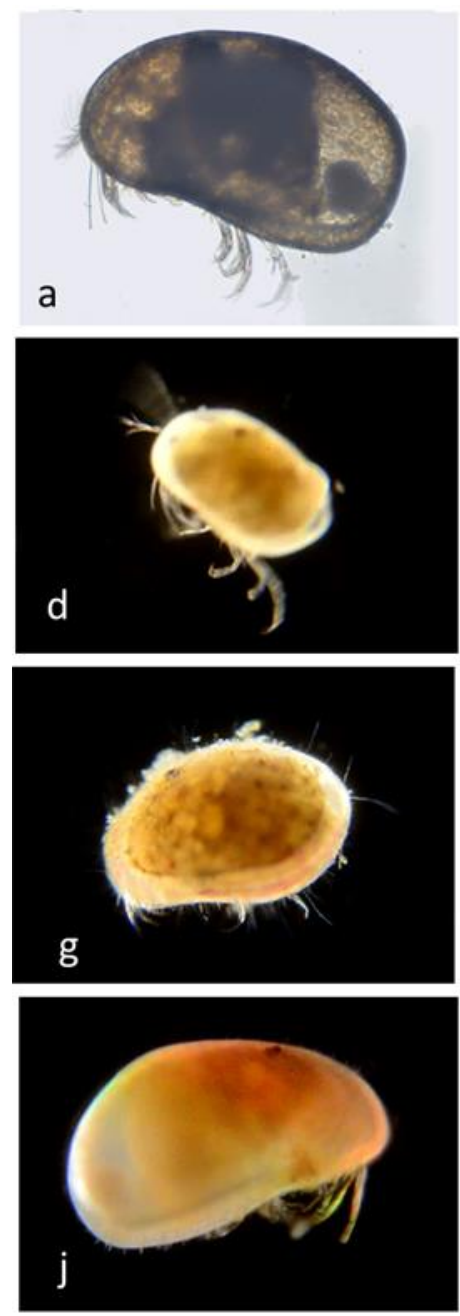
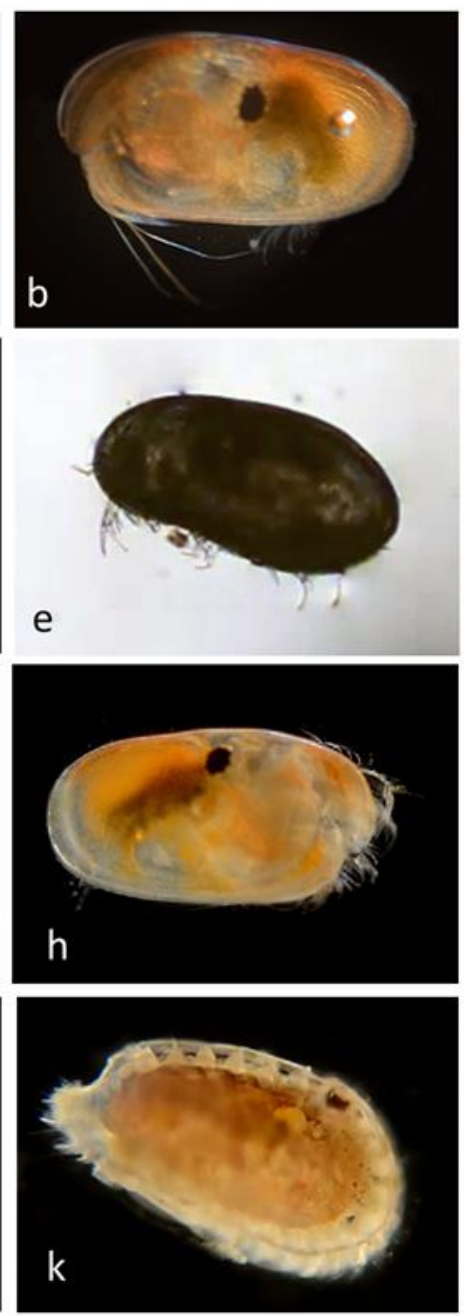
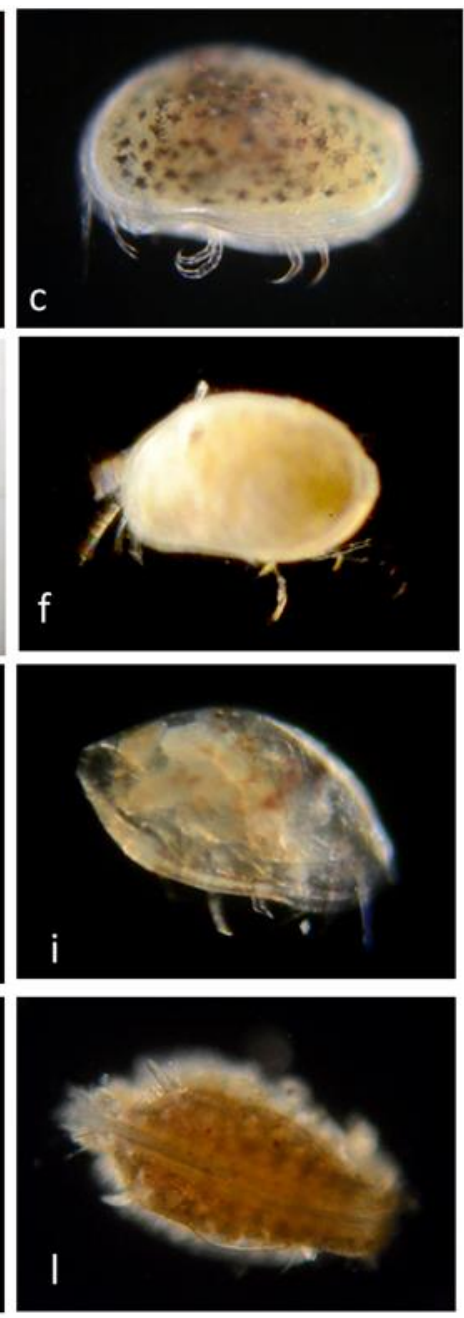

Fig. 12 Class Ostracoda. a, Podocopida (BIN ACV5505; n=1) BIOUG01212-E01 from Upper Newport Bay. b, BIN (BIN ACY8039; n=1) BIOUG19282-F05 from Crew Dock, Upper Newport Bay. c, Podocopida (BIN ACV4889; n=1) BIOUG01212-A06 from Delhi Channel, Upper Newport Bay. d, Podocopida (BIN ACU2544; n=1) BIOUG01226-H04 from Catalina Island harbor. e, Podocopida (BIN ACK9323; n=2) BIOUG01207-D03 from Balboa Island at Coral Street. f, (n=2) CCDB-32335 A08 from Balboa Island at Coral Street. g, (n=1) CCDB-25430 B07 from Crew Dock, Back Bay Science Center. h, (n=1) CCDB-24003 C07 from Balboa Island at Coral Street. i, (n=1) CCDB-32335 H02 from Balboa Island at Coral Street. j, (n=1) CCDB-31779 C11 from Shaw's Cove, Laguna Beach. k, Podocopida (BIN ACN1418; n=3) BIOUG19282-A05 from Balboa Island at Crystal Street. 1, Podocopida (BIN ACN1418; n=3) BIOUG01218 D09 from Balboa Island at Coral Street. 


\section{Class Malacostraca}

This is the largest of the six classes of crustacea, containing about 40,000 living species divided among 16 orders including crabs, lobsters, crayfish, shrimp, krill, woodlice, amphipods, mantis shrimp and other animals [23]

Order Amphipoda. Only two of the amphipods described here can be identified to species, but 23 forms were shown to be distinguishable by DNA barcode (Figure 13).
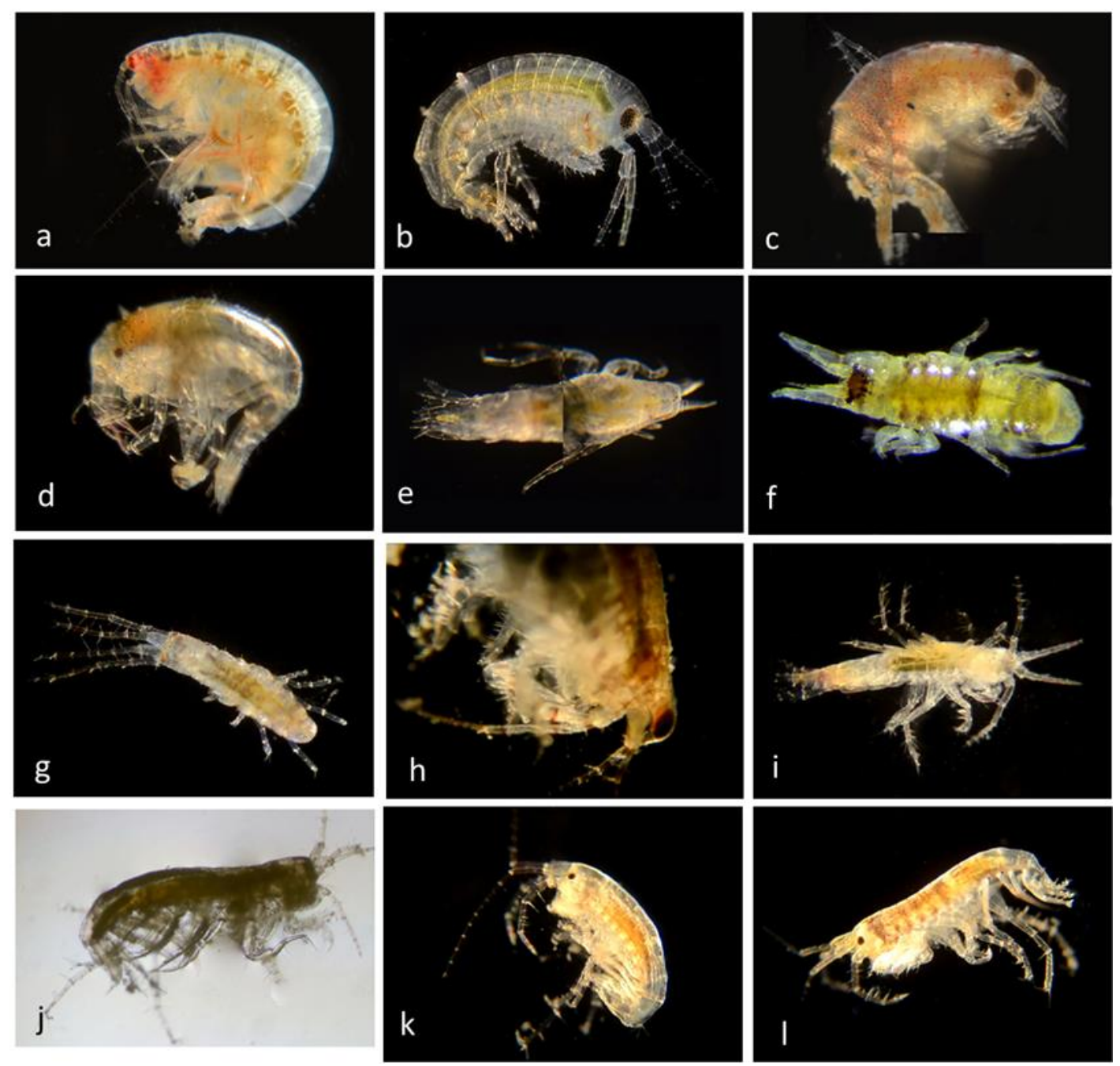

Fig. 13 Class Amphipoda. a-e, Family Ampeliscidae. a, Ampelisca agassizi [16](BIN ACH03884; n=1) BIOUG01218 F08 from off Dana Point. b, Family Ampeliscidae (BIN ADP8184; n=3) CCDB-31777 E04 from Laguna Beach. c, Family Ampeliscidae (BIN ACN1096; n=3) BIOUG19282-H09 from Balboa Island at Coral. d, Family Ampeliscidae (no BIN) CCDB-32335 E07 from Back Bay Science Center dock. e, Family Ampeliscidae (BIN ACM3513; n=1) BIOUG01212 D04 from off Dana Point. f, Family Corophiidae. Laticorophium baconi [16](BIN AAH4089, n=4) BIOUG19287 G05 from Balboa at Coral. g, Family Ischyroceridae (BIN ACK9657; n=4) CCDB-31777 G08 from Balboa at Coral. h, (BIN ACN0148; n=1) BIOUG01218 D02 from Lido at Genoa, Newport Harbor. i, (BIN ACU0862; n=2) BIOUG01226 H10 from Newport Pier. j, (BIN ACK9023; n=2) BIOUG01207-F08 from Harbor Patrol Dock, Newport Harbor. k, (BIN ACM3353; n=2) BIOUG01212-D06 from Back Bay Science Center Dock. 1, (BIN ACM3732; n=1) BIOUG01212-D09 from Back Bay Science Center Dock. 

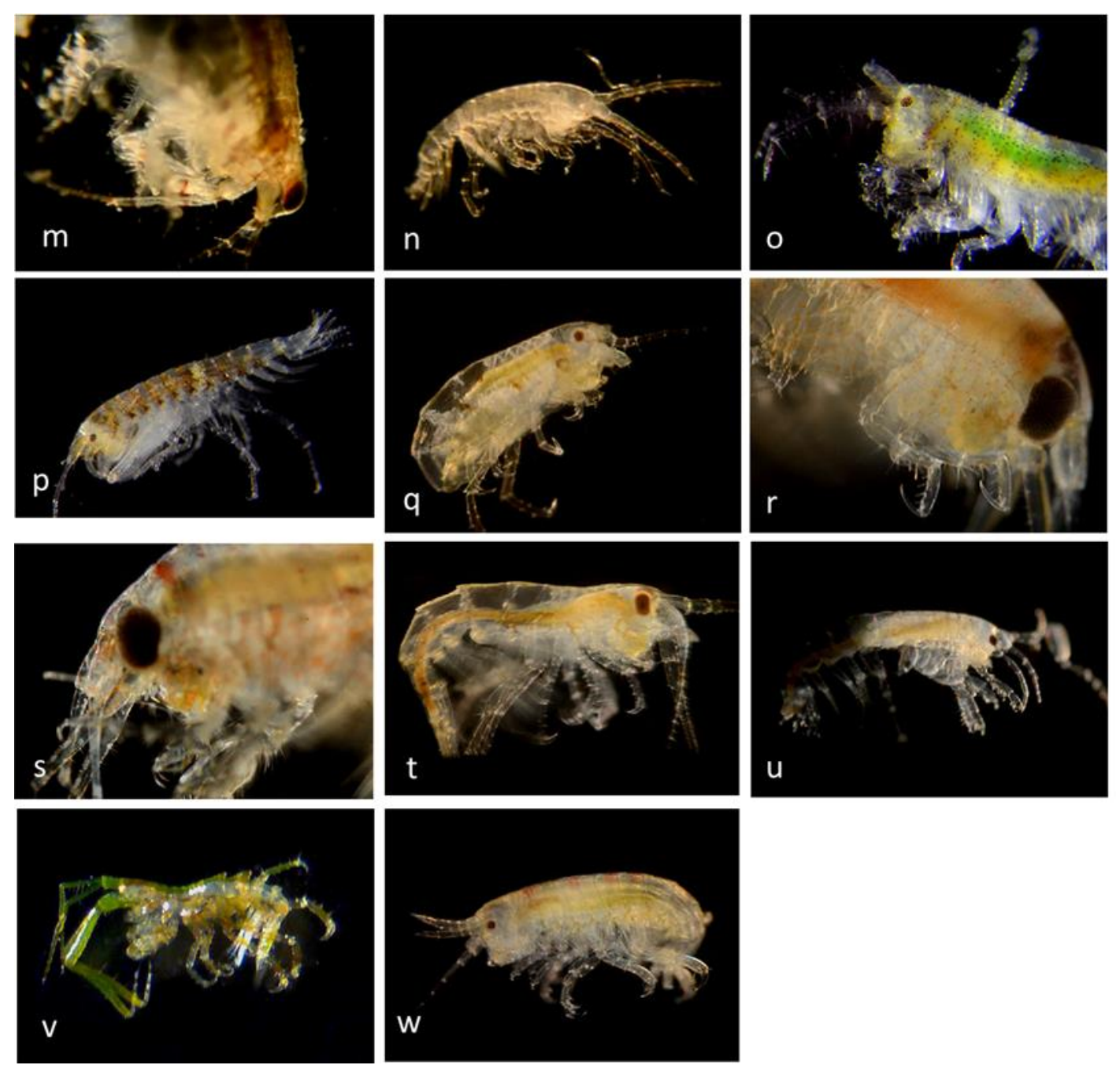

Fig. 13 Class Amphipoda, cont. m, (BIN ACN0148; n=1) BIOUG01218 D02 from Lido at Genoa. n, (BIN ADM8060; n=1) CCDB 24625 F07 from Bahia de Los Angeles, Baja California. o, (no BIN) CCDB-25430 A12 from Balboa at Coral. p, (no BIN) CCDB-24003 F12 from Crew Dock, Upper Newport Bay. q, (no BIN) CCDB-24003 F09 from Balboa at Coral. r, (no BIN) CCDB-24003 D09 from Balboa at Coral. s, (no BIN) CCDB-24003 C06 from Balboa at Coral. $\mathbf{t}$, (no BIN) CCDB-24003 C04 from Balboa at Coral. $\mathbf{u}$, (no BIN) CCDB-24003 A04 from Balboa at Coral. v, (no BIN) BIOUG19287 G07 from Bahia de Los Angeles, Baja California. w, Family Hyalidae (no BIN) CCDB-31779 H06 from Shaw`s Cove, Laguna Beach.

Family Caprellidae (skeleton shrimps). Although these animals are typically found attached to a substrate (usually floating algae) they often detach during capture and so appear in our plankton samples. Our collection (Figure 14) includes representatives of five different unidentified species, according to DNA barcodes. Seventeen local species in this family have been listed [16] 

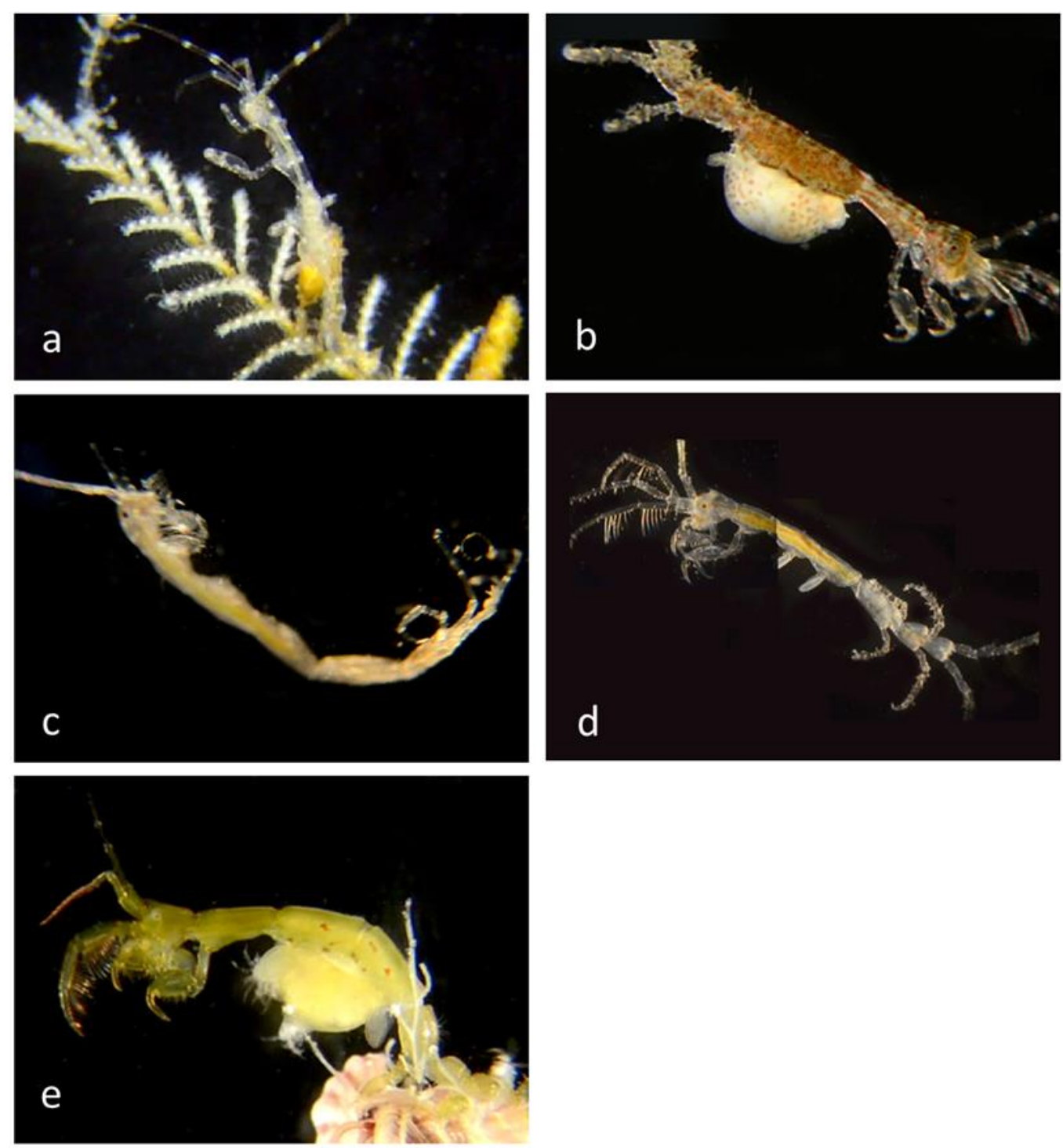

Fig. 14 Class Amphipoda; Family Caprellidae. a, Caprellidae (BIN ADM 9209; n=1) CCDB 24625 H07 from Bahia de Los Angeles. b, Caprella sp. (BIN ACN0028; n=1) BIOUG01218 G12 from Balboa at Coral. c, Caprella sp. (BIN ACK9550; n=6) BIOUG01218 D01 from Lido at Genoa. d, Caprella sp. (BIN ACN0029; n=2) BIOUG19282-H08 from Balboa at Coral. e, Caprella sp. (BIN ACU1090; n=5) BIOUG01226-H02 from Catalina Island (but another from Long Beach).

Order Cumacea [32]. Four different unidentified species, according to DNA barcodes (Figure 15) were collected. 53 local species in this order have been listed [16] 

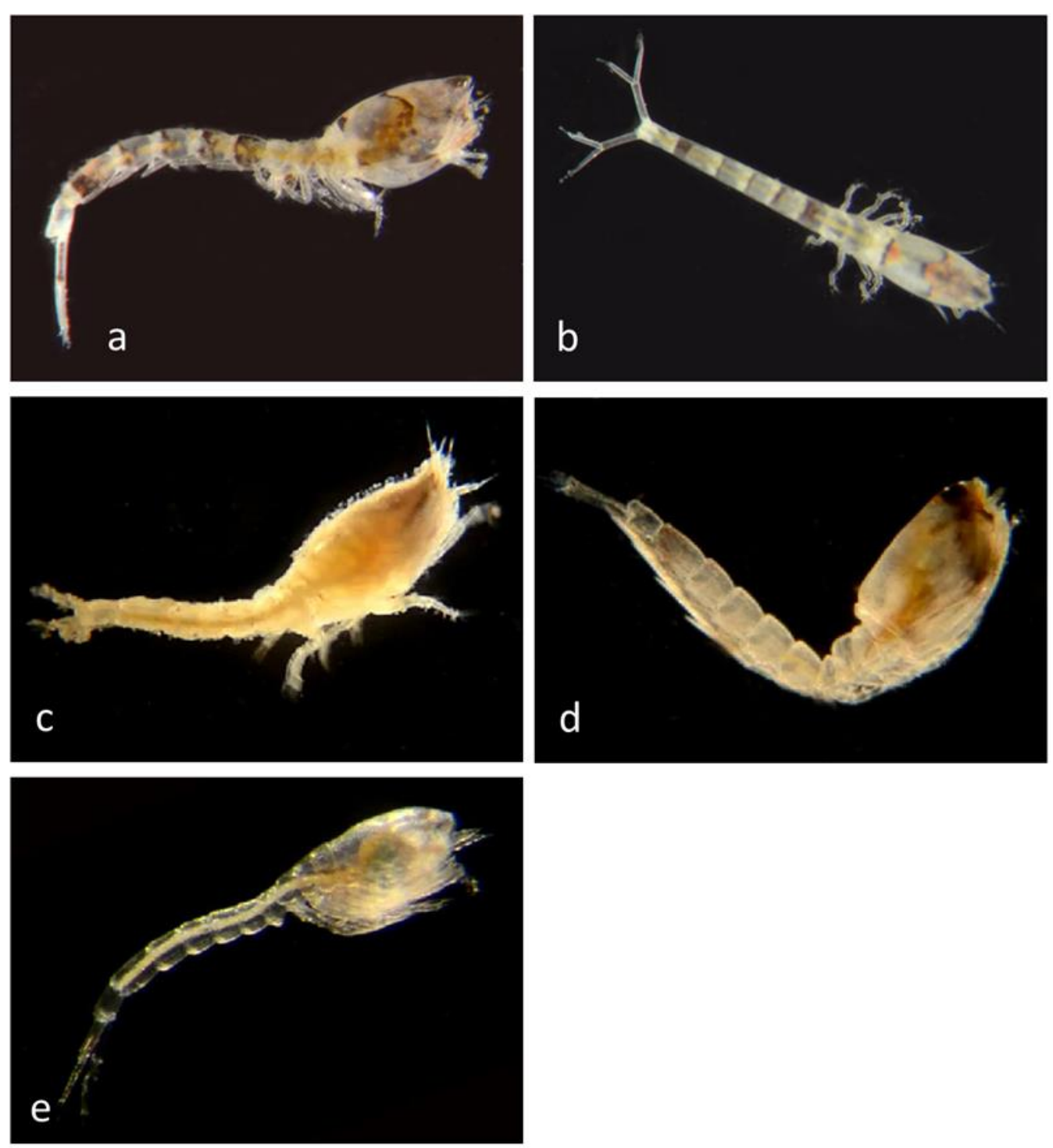

Fig. 15 Class Malacostraca; Order Cumacea. a, (BIN ADM7441; $n=4$ ) CCDB 24625 G08 from Bahia de Los Angeles. b, (BIN ADM7441; n=4) CCDB 24625 G07 from Bahia de Los Angeles. c, (BIN ACY8449; n=1) BIOUG19282-A06 from Balboa at Crystal. d, (BIN ACU1366; n=1) BIOUG01226-F08 off Newport Pier. e, (BIN ACU1019; n=1) BIOUG01226A06 off Newport Pier.

\section{Order Decapoda}

Many decapods go through early planktonic stages, following the nauplius, called zoea and megalops [9]. The following families are represented in the collection:

Family Alpheidae: Individuals of two unidentified species were collected in Bahia de Los Angeles (Figure 16a,b). Ten local species in this family have been listed [16].

Family Blepharopodidae: One zoea larva of Blepharipoda occidentalis [16] was collected and identified by DNA barcode, and an adult, recognized morphologically, was also collected (Figure 16c,d).

Family Callianassidae: One adult of Neotrypaea californiensis [16] that was purchased from a dealer, and a juvenile of a different species, were identified by DNA barcode (Figure 16e,f). 

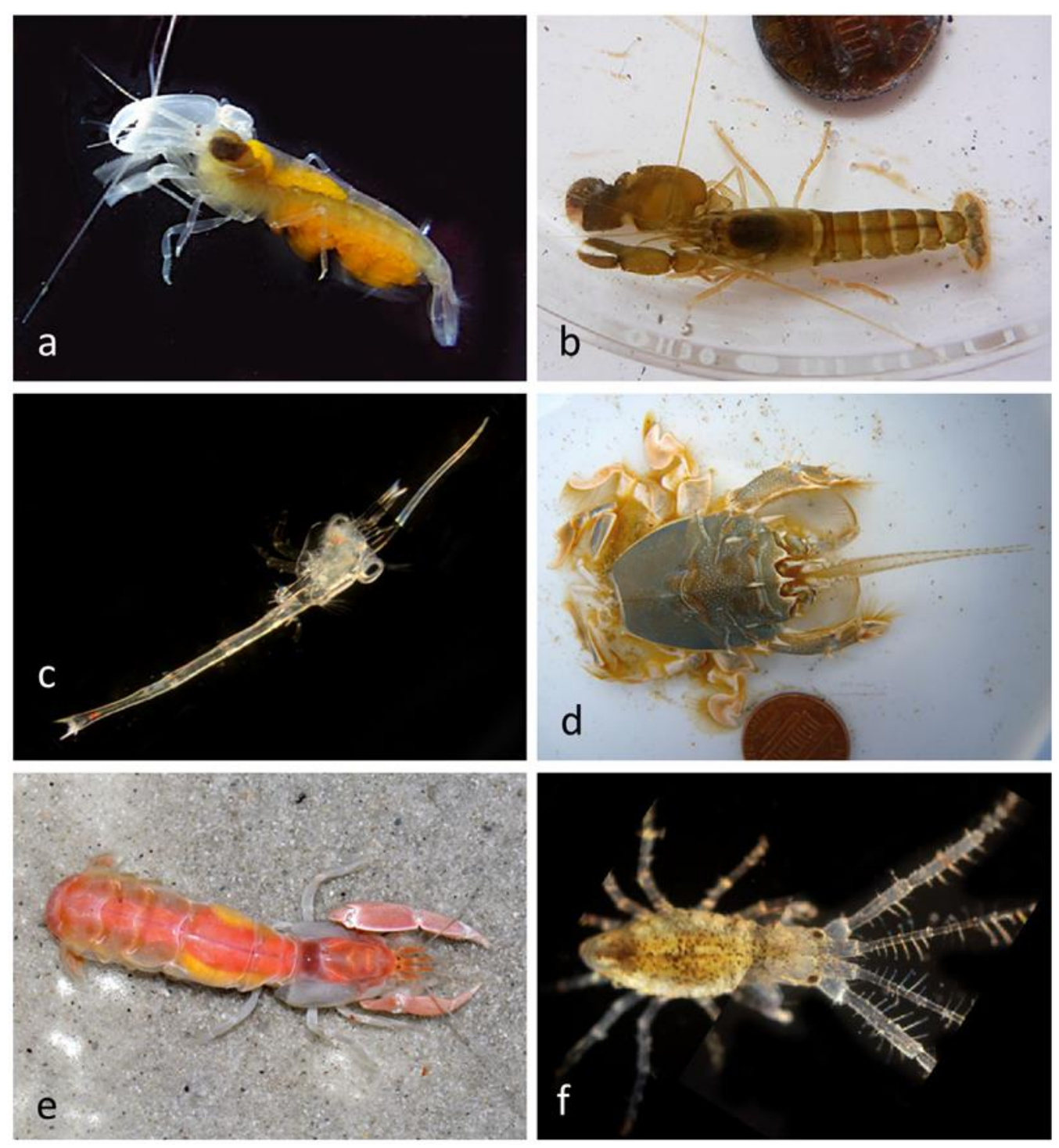

Fig. 16 Order Decapoda. a,b, Family Alpheidae. a, (BIN ADM7299; n=1 adult) CCDB 24625 G02 from Bahia de los Angeles. b, (No DNA sequence) BIOUG19287 D07 from Bahia de los Angeles. c,d, Family Blepharipodidae. c, Blepharipoda occidentalis (BIN AAW7488; n= 3 zoeae) Zoea larva CCDB 24622 C12 from Newport Harbor entrance. $d$, Blepharipoda occidentalis (No DNA sequence) from Crystal Cove State Park, Orange County, CA. e,f, Family Callianassidae. e, Neotrypaea californiensis [16](BIN ACA5392; n=1) BIOUG01212-C10 Purchased from Big Fish, Seal Beach, who purchased it from Washington State. f, Callianassidae (BIN ADT1830; n=1) CCDB 24622 F12 from Balboa at Coral.

Family Epialtidae: Two zoea larvae and one adult of the Sheep Crab Loxorhynchus grandis [16] were matched by DNA barcoding (Figure 17). Surprisingly, some Mysid shrimp (Figure 17e,f) also gave a DNA barcode corresponding to Loxorhynchus grandis. It seems likely that this reflects gut contents, rather than body tissue of these specimens. 

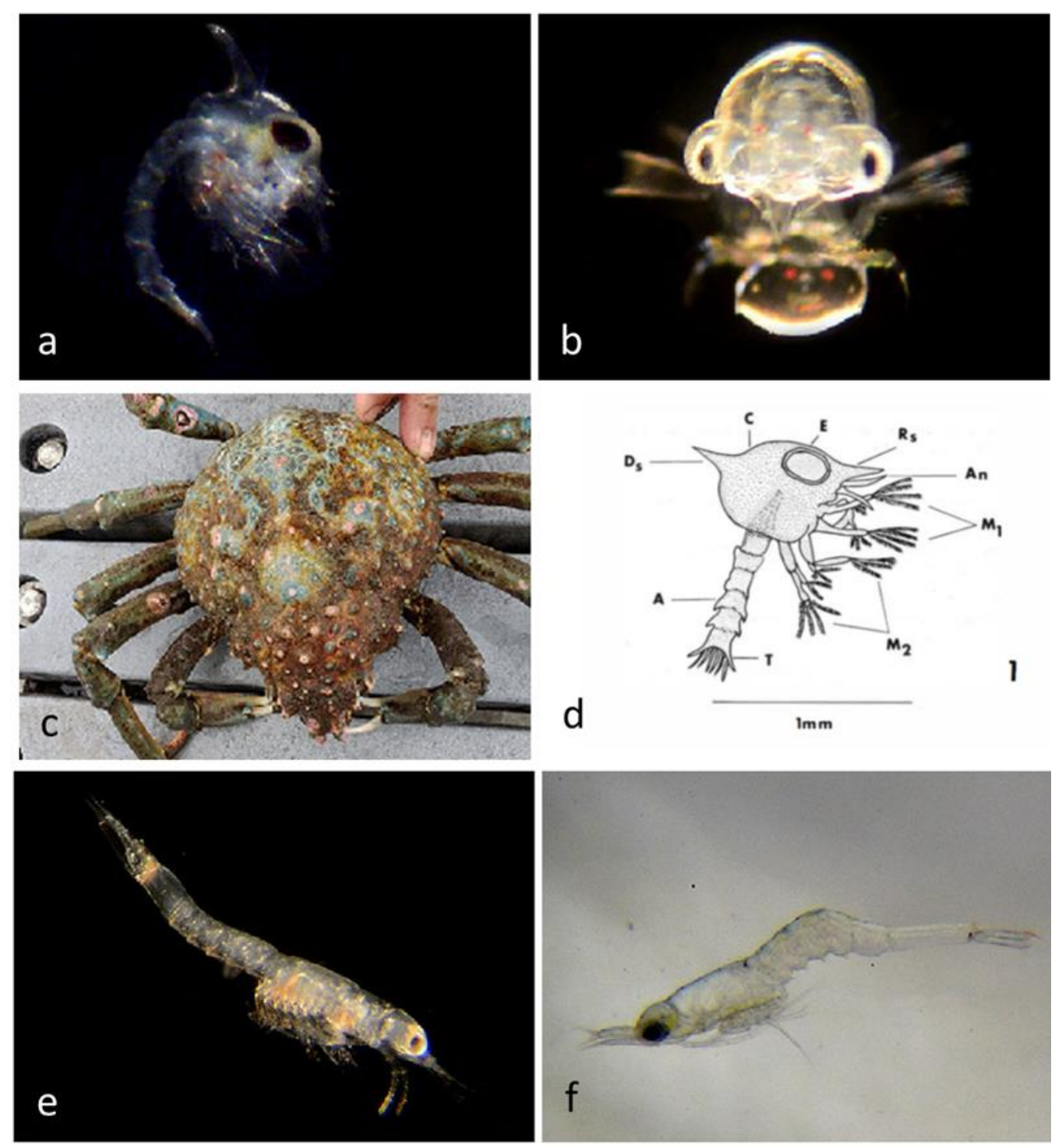

Fig. 17 Order Decapoda; Family Epialtidae. Loxorhynchus grandis (Sheep Crab) BIN AAX6597 (n= 2 zoeae, 1 adult). a, Zoea BIOUG19287 G04 from Bahia de Los Angeles, Baja California. b, Zoea BIOUG19287 G12 from Ocean off Newport Beach. c, Adult BIOUG19287 D02 off Balboa Pier, Newport Beach. d, Nomenclature of zoea appendages. e, Mysid shrimp BIOUG19287 A02 from Newport Harbor entrance giving DNA barcode in BIN AAX6597. f, Mysid shrimp BIOUG19287 H07 from offshore Orange County, CA. giving DNA barcode in BIN AAX6597.

Also in this family, one zoea larva of Pelia tumida (Dwarf Teardrop Crab) [16] was matched by DNA barcoding to an adult with a published DNA barcode from the Natural History Museum of Los Angeles County (Figure 18a,b). One zoea larva and one adult of Pugettia dalli (Spined Kelp Crab) [16] were matched by DNA barcode (Figure 18c,d), and one adult of Eriphia granulosa from Bahia de Los Angeles (Figure 18e) was barcoded. 

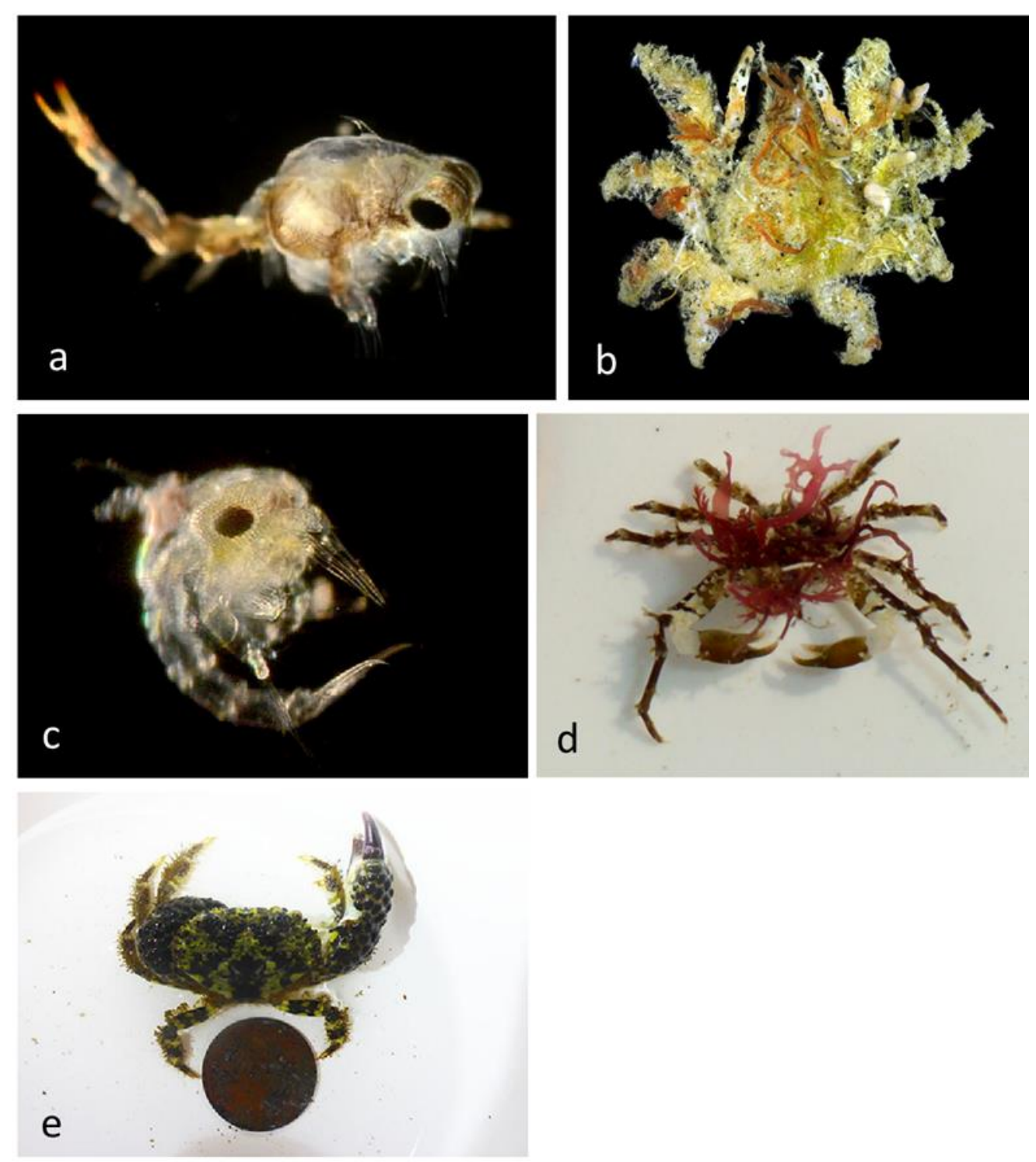

Fig. 18 Order Decapoda: a-d Family Epialtidae. a,b, Pelia tumida (BIN ADP0347; n=1 zoea) (Dwarf Teardrop Crab). a, Zoea larva CCDB 24622 D08. Newport Beach Harbor Entrance. b, Adult. Natural History Museum of Los Angeles County. c,d, Pugettia dalli (BIN AAX7214; n=1) (Spined Kelp Crab). c, Zoea CCDB-32335 C05. Newport Beach, Balboa at Coral. d, Adult. Little Treasure Cove, Crystal Cove State Park. e, Family Eriphiidae. Eriphia granulosa (BIN ACH8870; n=1) Adult BIOUG19287 D10 at Bahia de Los Angeles.

Family Cancridae: Zoea and megalops larvae of the Yellow Rock Crab Metacarcinus anthonyi [16] were matched by DNA barcode and an adult image from Wikipedia is included in Figure 19. 

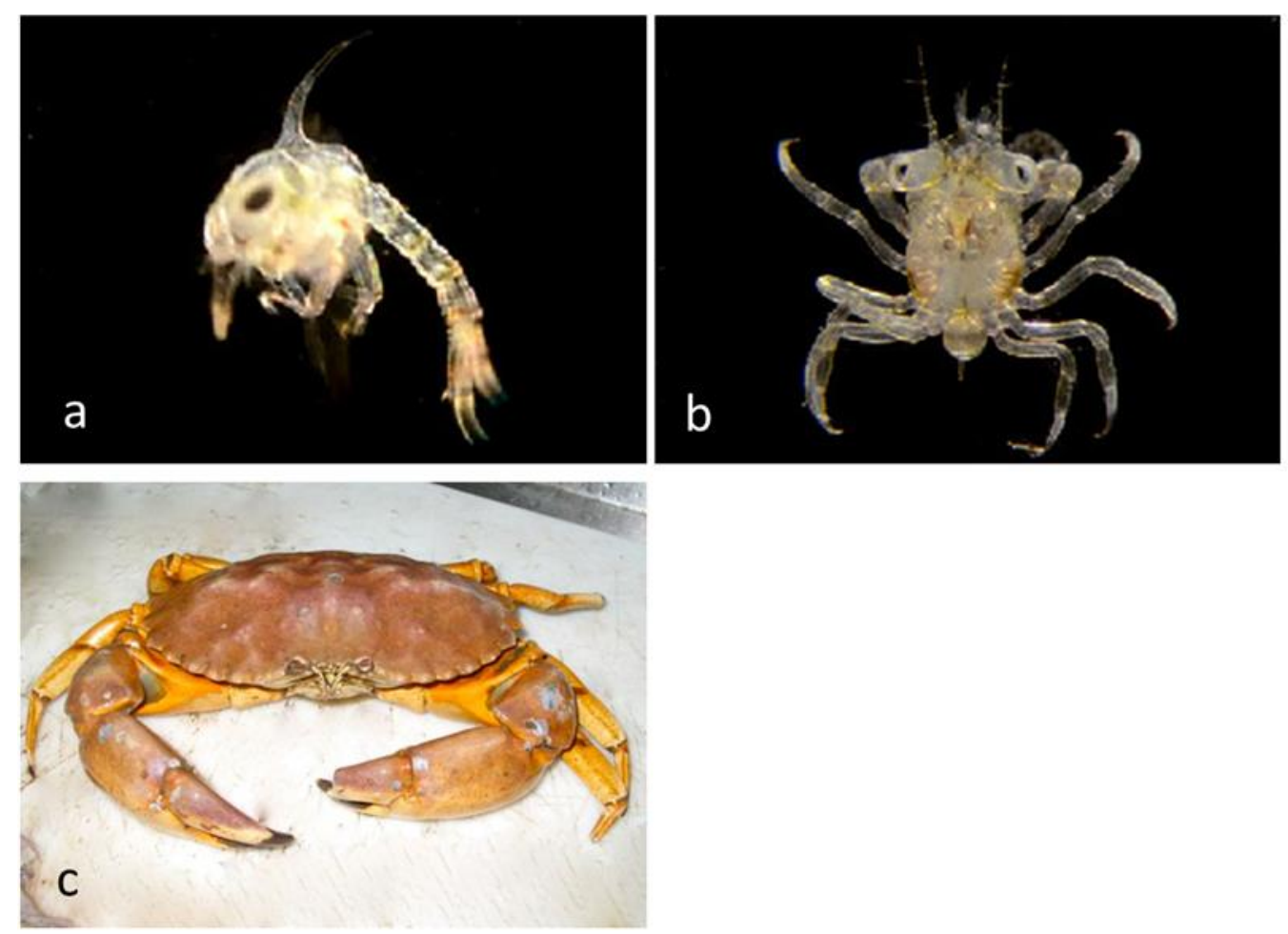

Fig. 19 Order Decapoda: Family Cancridae: Metacarcinus anthonyi (BIN AAX3827; n=1 zoea, 2 megalops) (Yellow Rock Crab). a, Zoea CCDB 24622 D11 from Newport Harbor entrance. b, Megalops CCDB-19281 F11 from Pacific Ocean off Newport Beach. c, Adult @ Ken Jones (from: Pier Fishing in California https://www.pierfishing.com/category/crustaceans-crabs-lobsters/).

Also in this family, a megalops larva of the Graceful Rock Crab Metacarcinus gracilis [16] was identified by DNA barcode and is shown with an image of the adult in Figure 20.
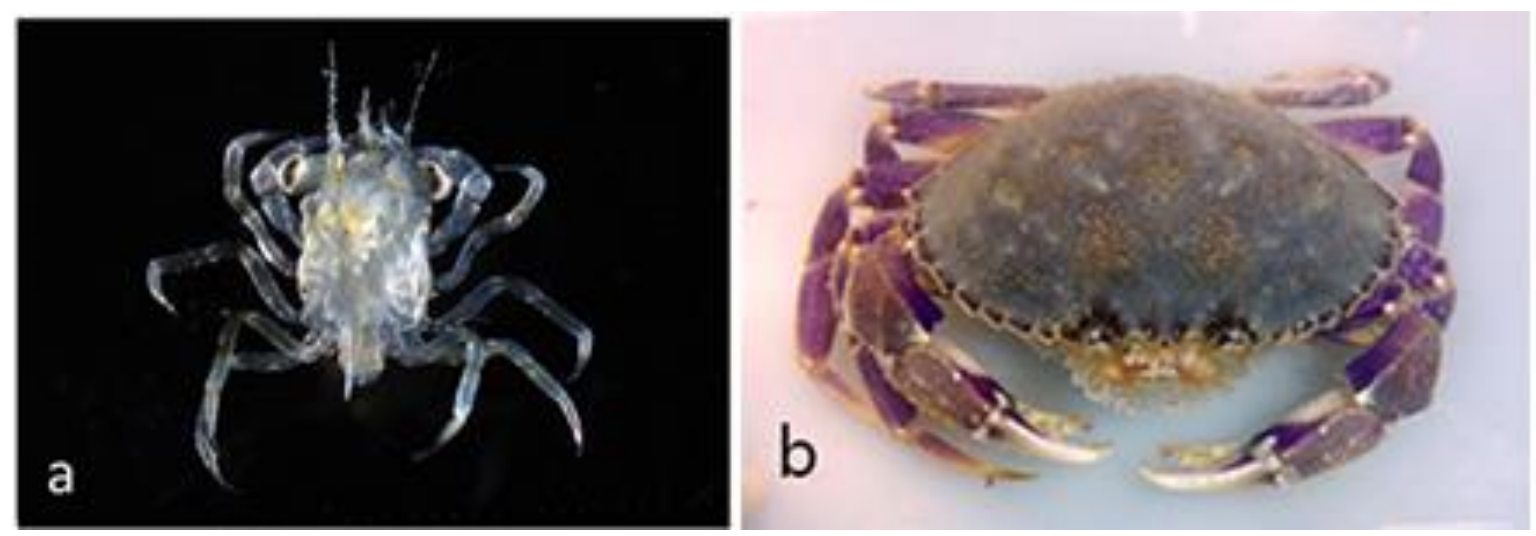

Fig. 20 Order Decapoda: Family Cancridae: Metacarcinus gracilis (BIN AAX3830; n=1 megalops) Graceful Rock Crab. a, BIOUG01218 D05 Megalops from Pacific Ocean off Dana Point. b, adult from San Francisco Bay (C Robin Gwen Agarwal. 
Family Grapsidae: The Lined Shore Crab Pachygrapsus crassipes [16] is one of the commonest intertidal decapods in our area. Zoea, megalops, and adult stages were matched by DNA barcoding (Figure 21).
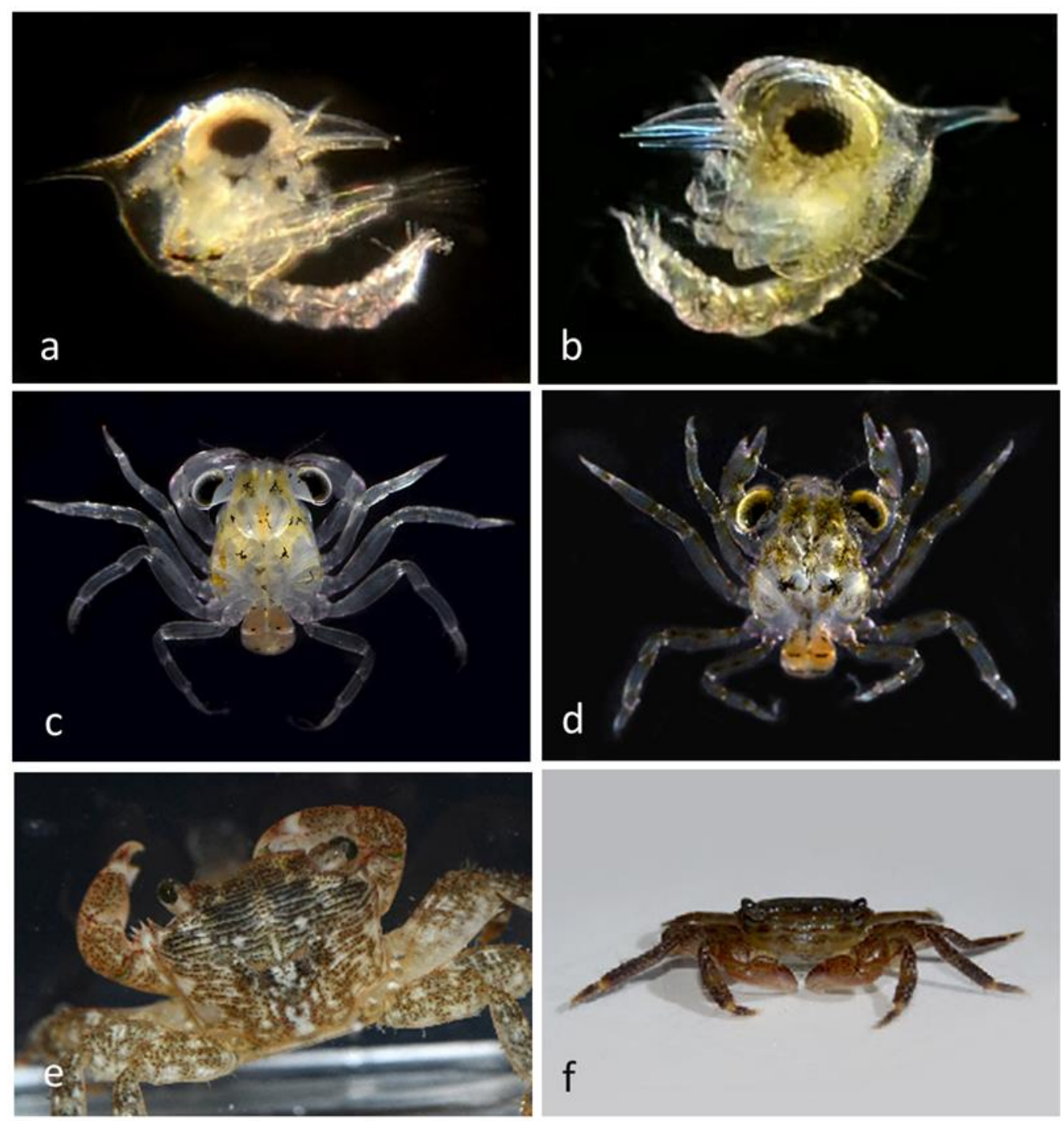

Fig. 21 Order Decapoda: Family Grapsidae: Pachygrapsus crassipes (BIN ACI1743; n= 6 adults; 12 megalops, 12 zoeae) Lined Shore Crab. a, Zoea BIOUG19282-G04 off Newport Aquatic Center. b, Zoea CCDB 24625 A01 from Newport Beach harbor. c, Megalops BIOUG01207-E08 off Newport Aquatic Center. d, Megalops BIOUG01207-E10 off Newport Aquatic Center. E, Adult. f, Adult BIOUG01218 C11 off Back Bay Science Center dock.

Family Hippidae: Although larval stages were not found, the DNA barcode of the common intertidal sand crab Emerita analoga [16] was obtained, and an image of it is included in Figure 22. 


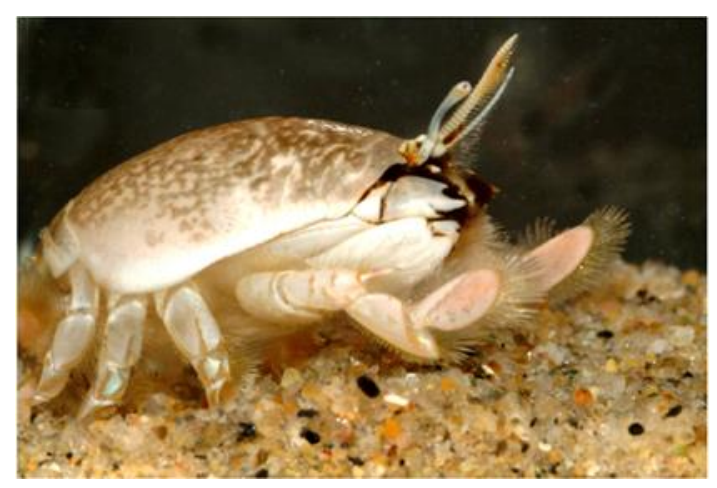

Fig. 22 Order Decapoda: Family Hippidae. Emerita analoga (BIN ACB4151; n=1) BIOUG01207-F04 Adult at Laguna Main Beach (No larvae found).

Family Hippolytidae: Two individuals of Hippolyte clarki [16](Figure 23 a,b) and one of Lysmata californica [16](Figure 23c) were identified by DNA barcoding.
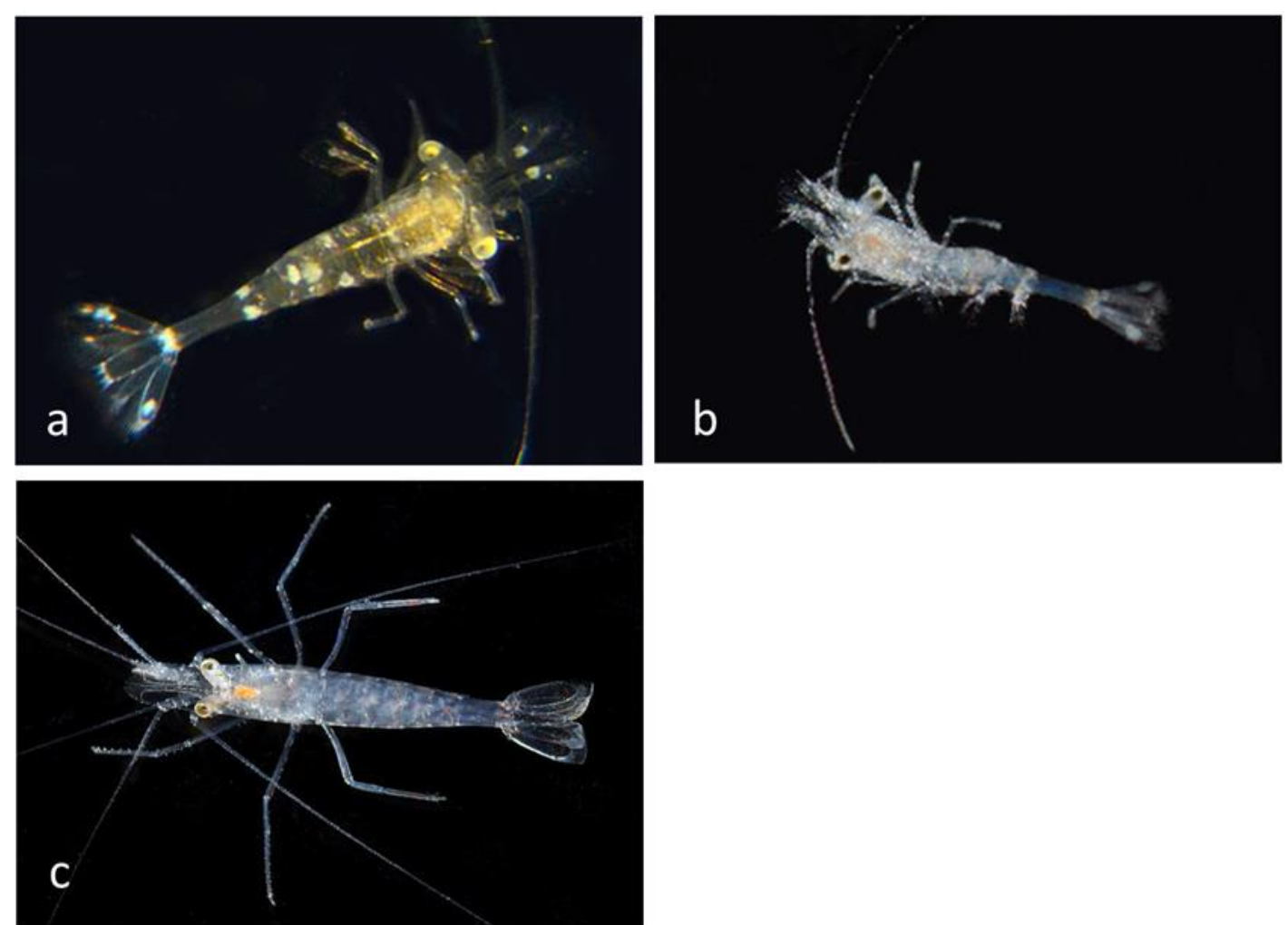

Fig. 23 Order Decapoda: Family Hippolytidae. a, Hippolyte clarki (BIN ACK9507; n=5) BIOUG19282-H05 Ocean off Crystal Cove. b, Hippolyte clarki (ACK9507) BIOUG01207-A08 Ocean off Crystal Cove. c, Lysmata californica (BIN ACN0141; n=1) BIOUG01218 D04 Ocean off Dana Point.

Family Muninidae: A larva of Pleuroncodes planipes [16] from Newport Harbor entrance was matched with an adult from Balboa at Crystal (Figure 24). 

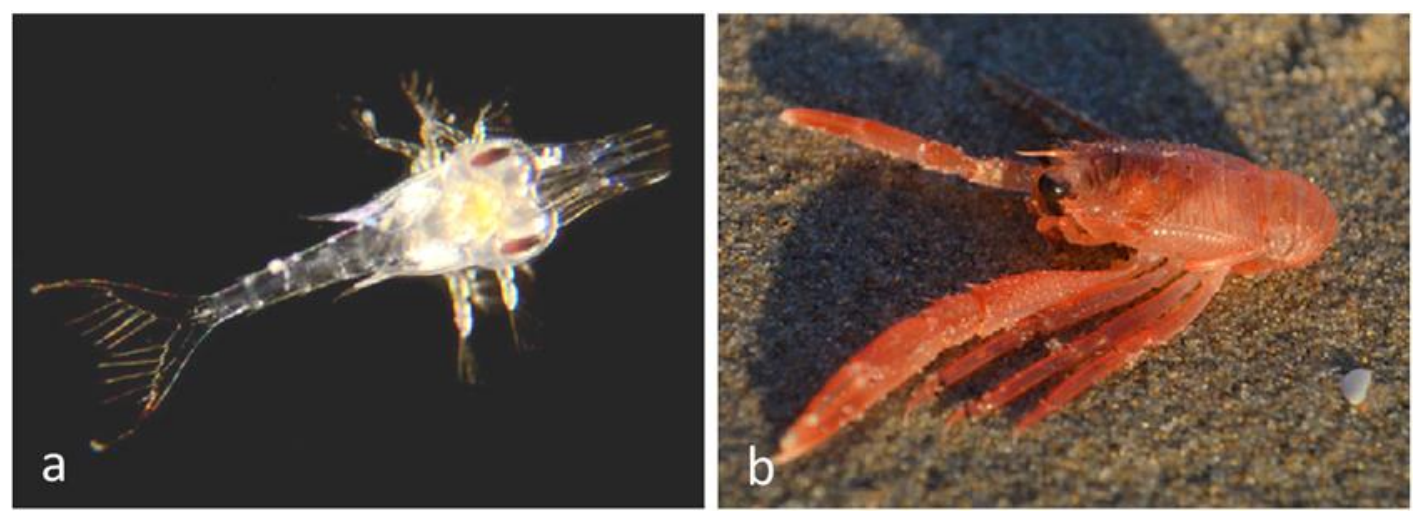

Fig. 24 Order Decapoda: Family Muninidae. Pleuroncodes planipes (BIN ACY 8753; n=1). a, Larva CCDB-31779 G07 from Newport Harbor Entrance. b, Adult BIOUG19282-A01 from Balboa at Crystal.

Family Ocypodidae: A juvenile of Leptuca crenulata (Crenulated Fiddler Crab)[16] at Shellmaker Island was matched with an adult from Bahia de Los Angeles, Baja California (Figure 25).
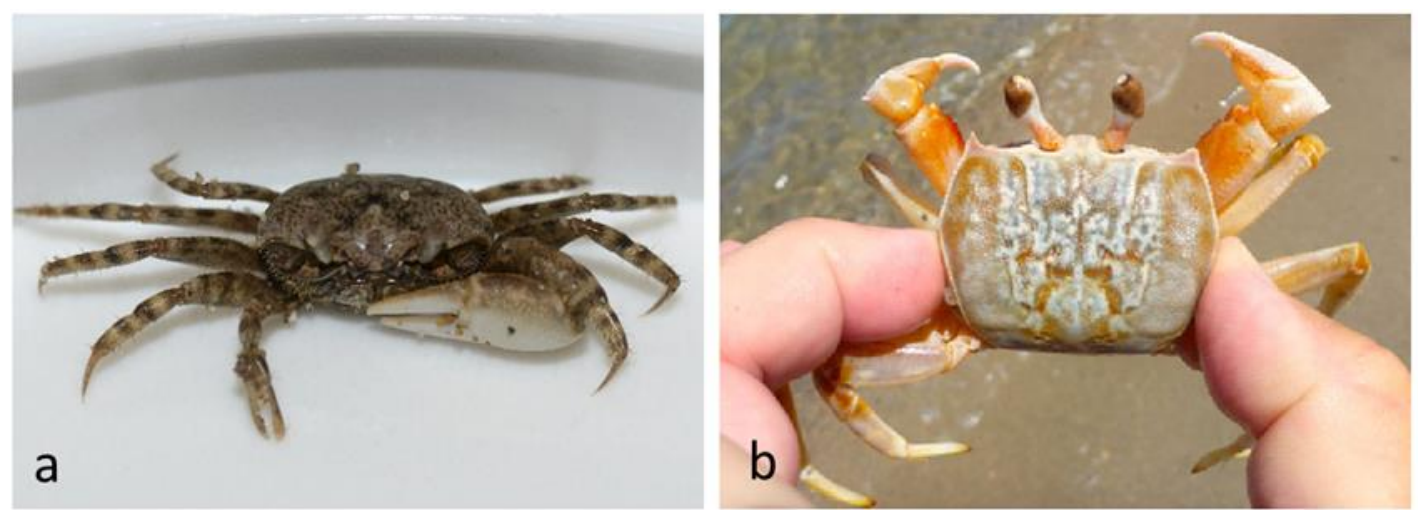

Fig. 25 Order Decapoda: Family Ocypodidae. Leptuca crenulata (BIN ACK 9146; n=3) Crenulated Fiddler Crab. a, Juvenile BIOUG01207-H07 at Shellmaker Island. b, Adult CCDB 24625 E05 at Bahia de Los Angeles, Baja California.

Ocypode occidentalis (Gulf Ghost Crab) at Bahia de Los Angeles, Baja California was identified by DNA barcode and morphology (Figure 26). 

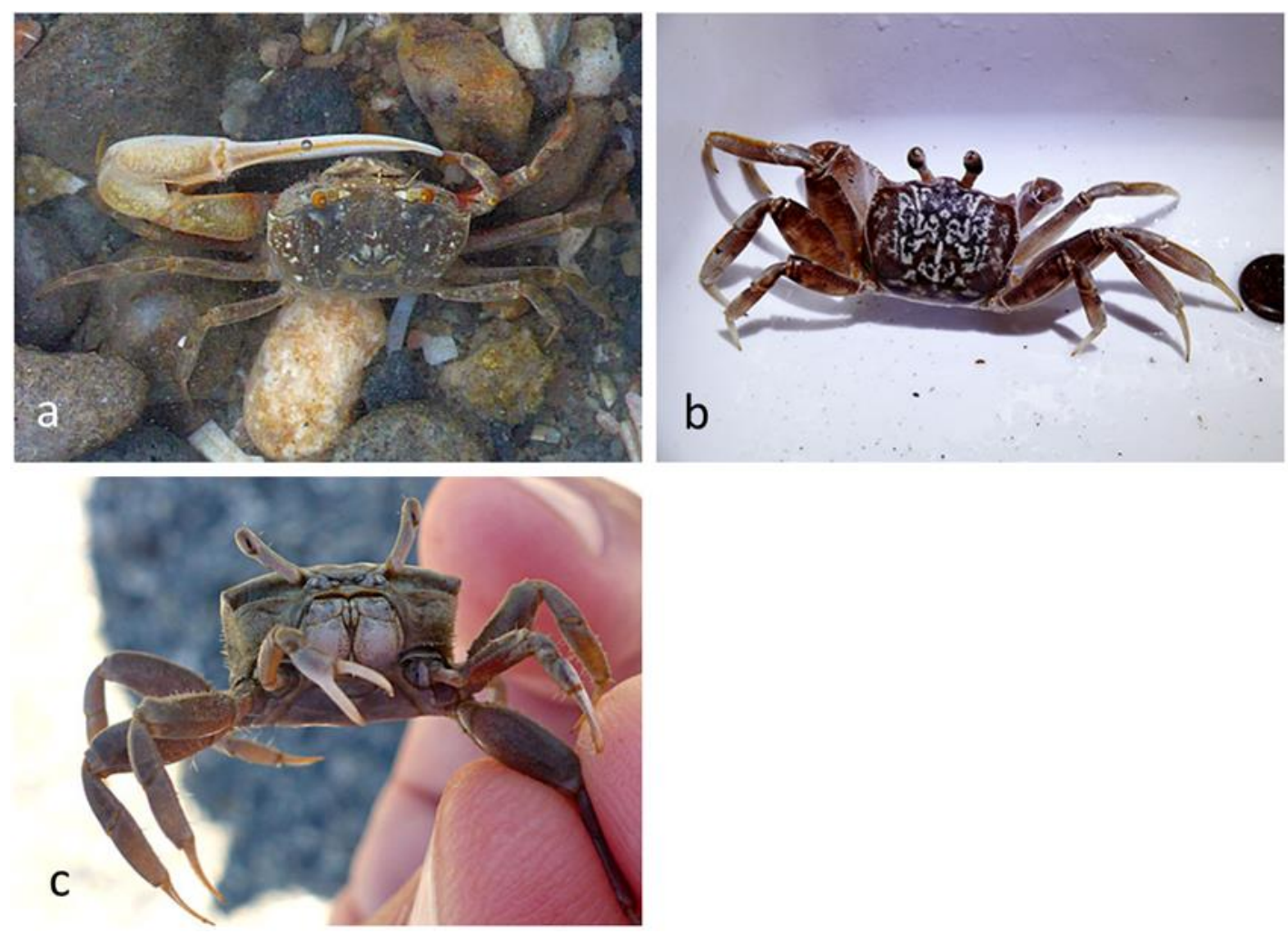

Fig. 26 Order Decapoda: Family Ocypodidae. Ocypode occidentalis (BIN ADK6959; n=2) Gulf Ghost Crab at Bahia de Los Angeles, Baja California. a, CCDB 24625 E03. b, BIOUG19287 D12 (not barcoded). c, CCDB 24625 E04.

Family Paguridae: An adult of Pagurus samuelis [16] from Crystal Cove State Park and a larva of Pagurus longicarpus [16] from Balboa at Coral were identified by DNA barcoding (Figure 27). Pagurus longicarpus (BIN ADQ3898) is reported from "along the Atlantic and Gulf coasts of the United States and the Atlantic coast of Canada." [16]
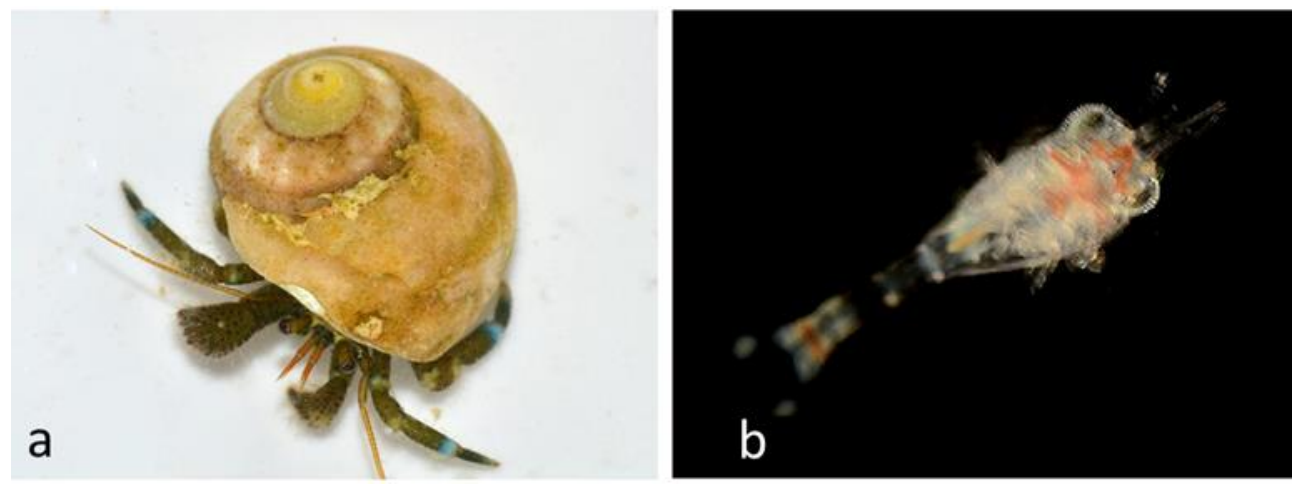

Fig. 27 Order Decapoda: Family Paguridae. a, Pagurus samuelis (BIN ABA9710; n=4) BIOUG01212-E11 from Crystal Cove State Park. b, Pagurus longicarpus (no BIN; n=1) larva CCDB-32335 B10 from Balboa at Coral.

Family Palaemonidae: Five unrecognized species were identified by DNA barcodes (Figure 28). 

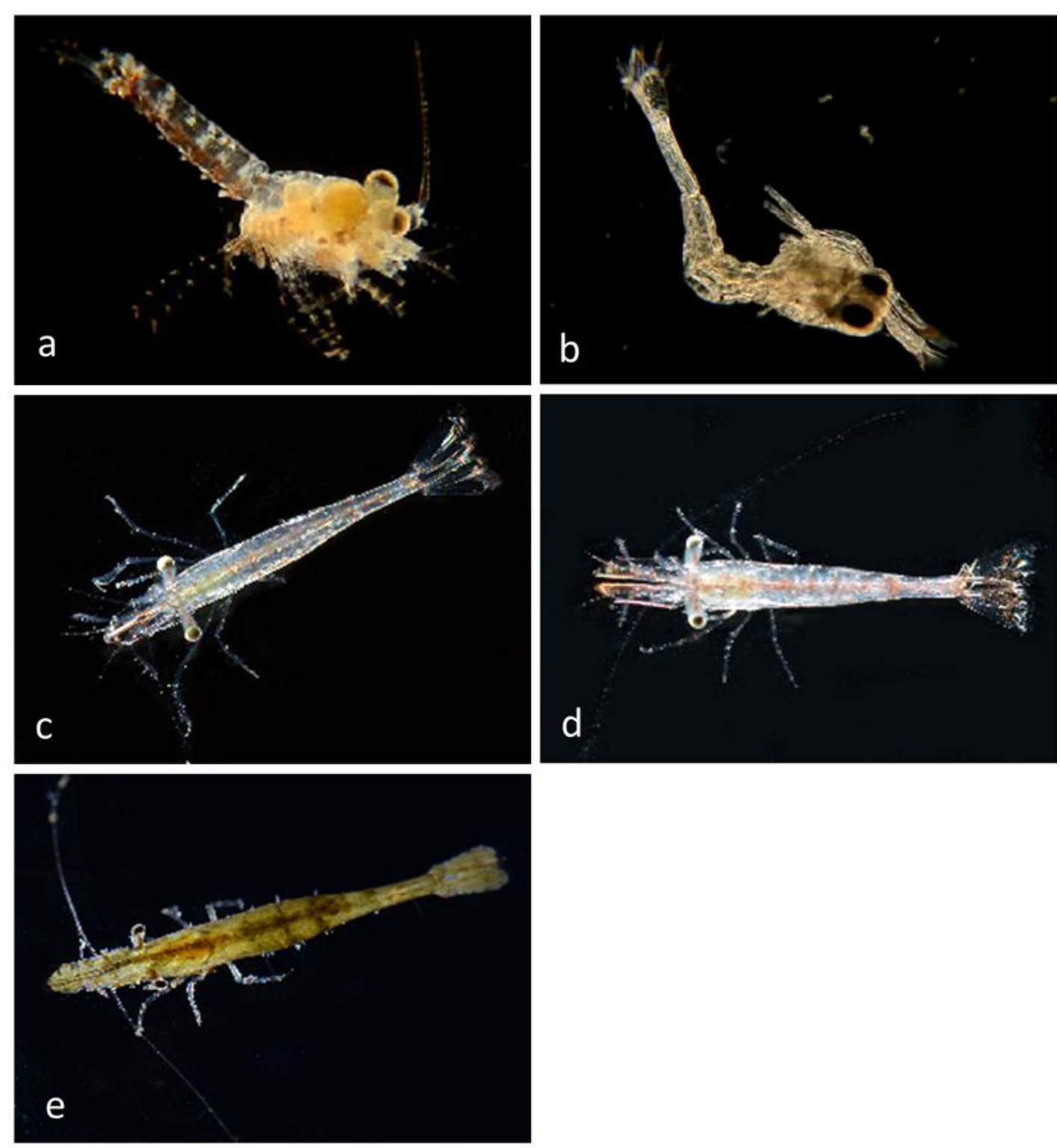

Fig. 28 Order Decapoda: Family Palaemonidae. a, (BIN ACN0140; n=6) BIOUG01226-G07 from Balboa at Coral. b, (BIN ACK9157; n=1) BIOUG01207-G05 off Newport Aquatic Center. c, (BIN ACK9412; n=15) BIOUG01218 H04 from Balboa at Coral. d, (BIN ACK9412; n=15) BIOUG01218 H05 from Balboa at Coral. e, (BIN ACK9412; n=15) BIOUG01218 A06 from Lido at Genoa.

Family Panopeidae: Lophopanopeus sp. zoea larvae (Figure 29 a,b) were matched with the adult (Figure 29c,d). Eurytium affine adults at Bahia de Los Angeles, Baja California and unidentified zoea from offshore at Newport Beach, CA. were barcoded (Figure 29 e-f). 

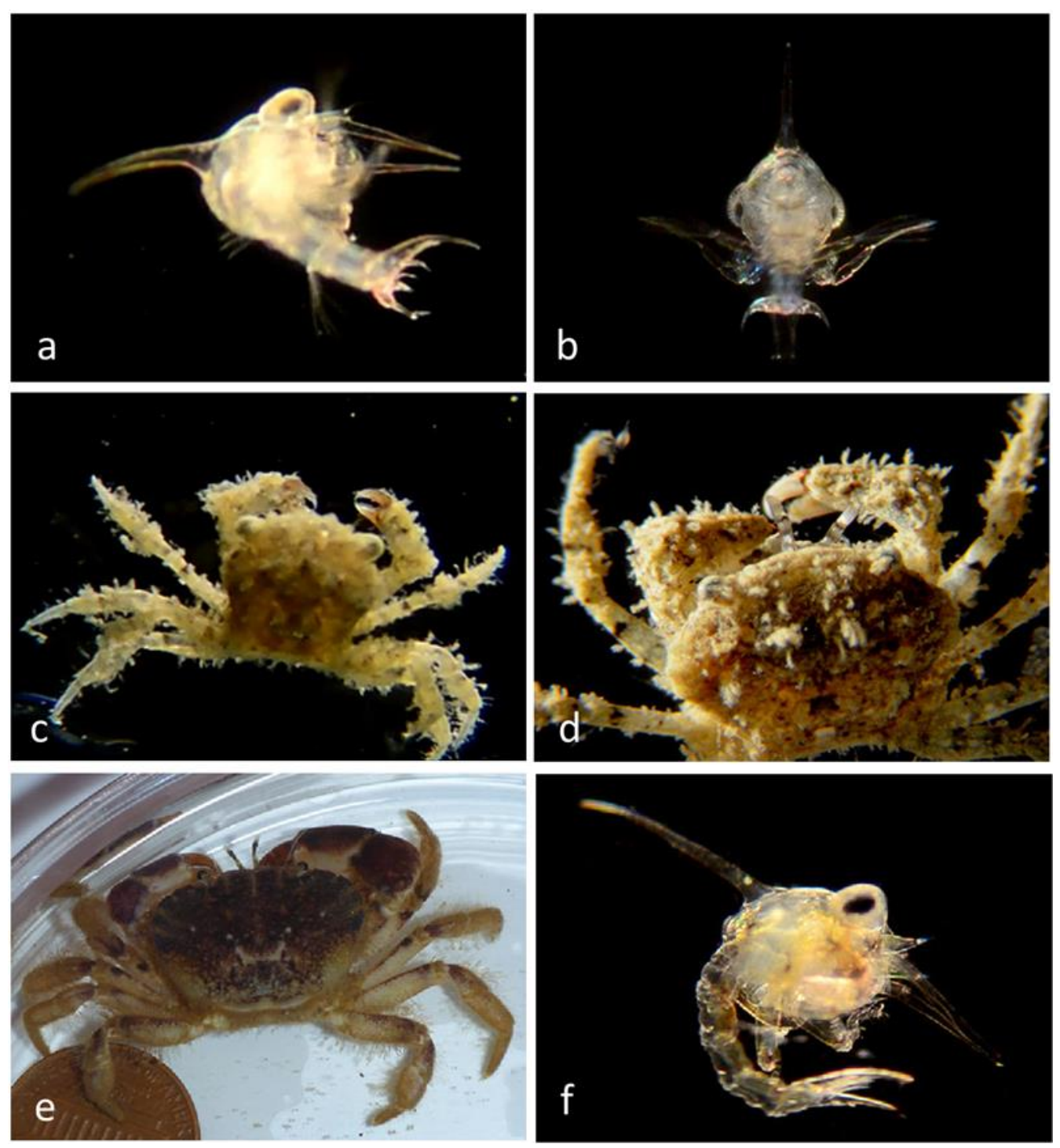

Fig. 29 Order Decapoda: Family Panopeidae: a-d. Lophopanopeus sp. (BIN ACK9404; n=2 zoeae plus 3 adults) a, Zoea BIOUG01226-H09 off Newport Aquatic Center. b, Zoea CCDB-32335 G06 from Balboa at Coral. c, Adult BIOUG19282D07 from Balboa at Coral. d, Adult CCDB-25430 B08 from Balboa at Coral. e, Eurytium affine (BIN ADM8008; n=2 adults) Adult CCDB 24625 G10 at Estacion del mar, Baja California. f, (BIN ACK9405; n=2 zoeae) Zoea BIOUG19287 H02 Offshore from Newport Beach, CA.

Family Penaidae: Farfantepenaeus californiensis [16] was collected from the Pacific Ocean off Crystal Cove and identified by DNA barcode (Figure 30).

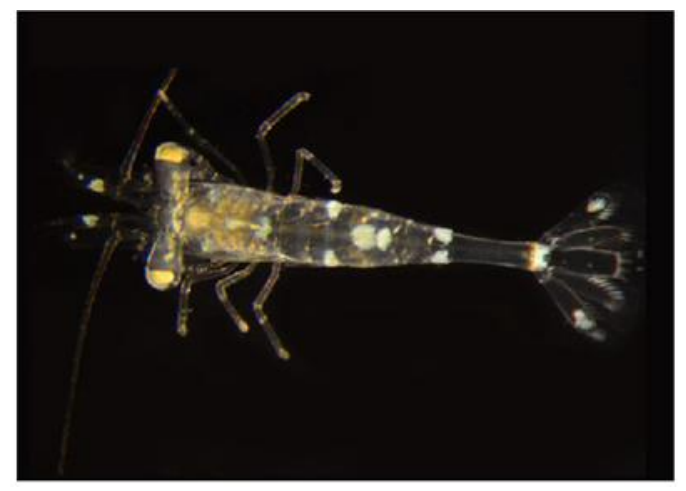


Fig. 30 Order Decapoda: Family Penaidae. Farfantepenaeus californiensis (BIN AAF7078; n=1). BIOUG19282-H04 from Ocean off Crystal Cove.

Family Pinnotheridae: Zoea larvae of the Pea crabs Pinnixa franciscana [16] and Pinnixa tubicola [16] were identified by DNA barcodes (Figure 31).

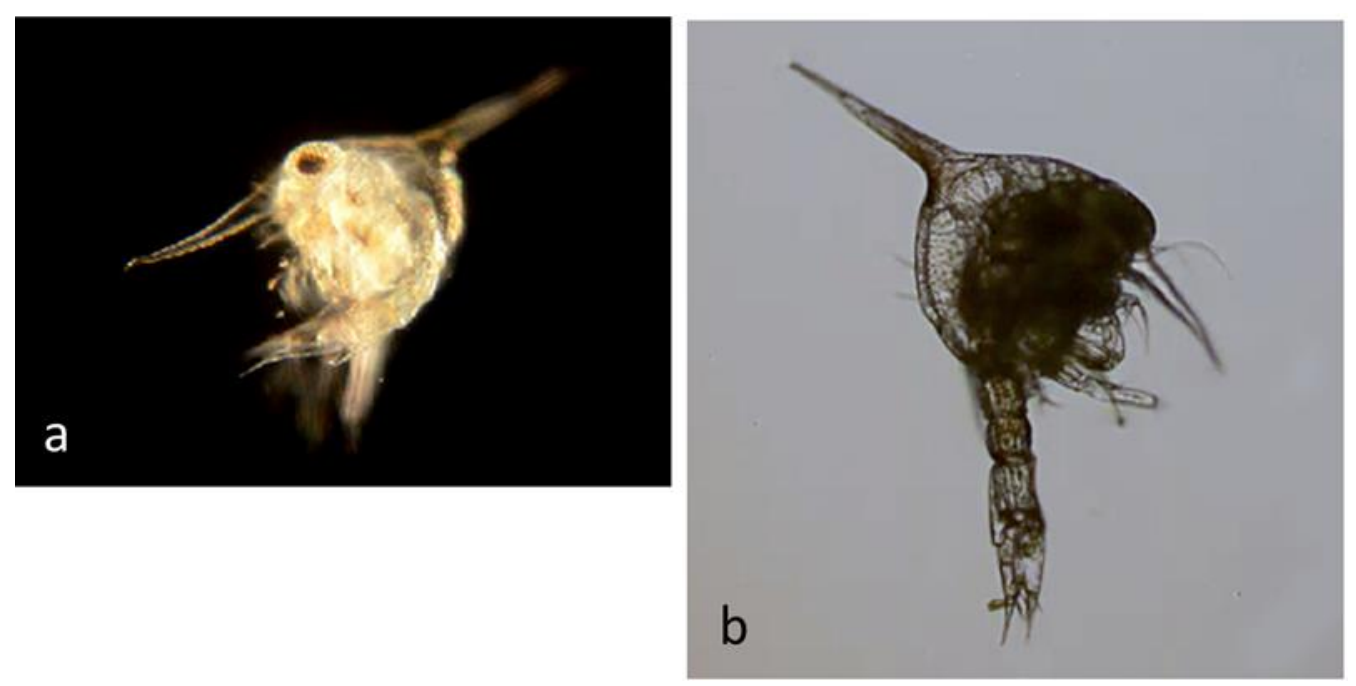

Fig. 31 Order Decapoda: Family Pinnotheridae. a, Pinnixa franciscana (BIN AAO4832; n=19 zoeae) Zoea larva CCDB32335 C02 from Balboa at Coral. b, Pinnixa tubicola (BIN ACK9533; n=1) Zoea larva BIOUG01207-B11 off Newport Aquatic Center.

Family Porcellanidae: Petrolisthes cabrilloi (Cabrillo's Porcelain Crab) zoea and adult, as well as zoea larvae from other members of this family were identified by DNA barcode (Figure 32). 

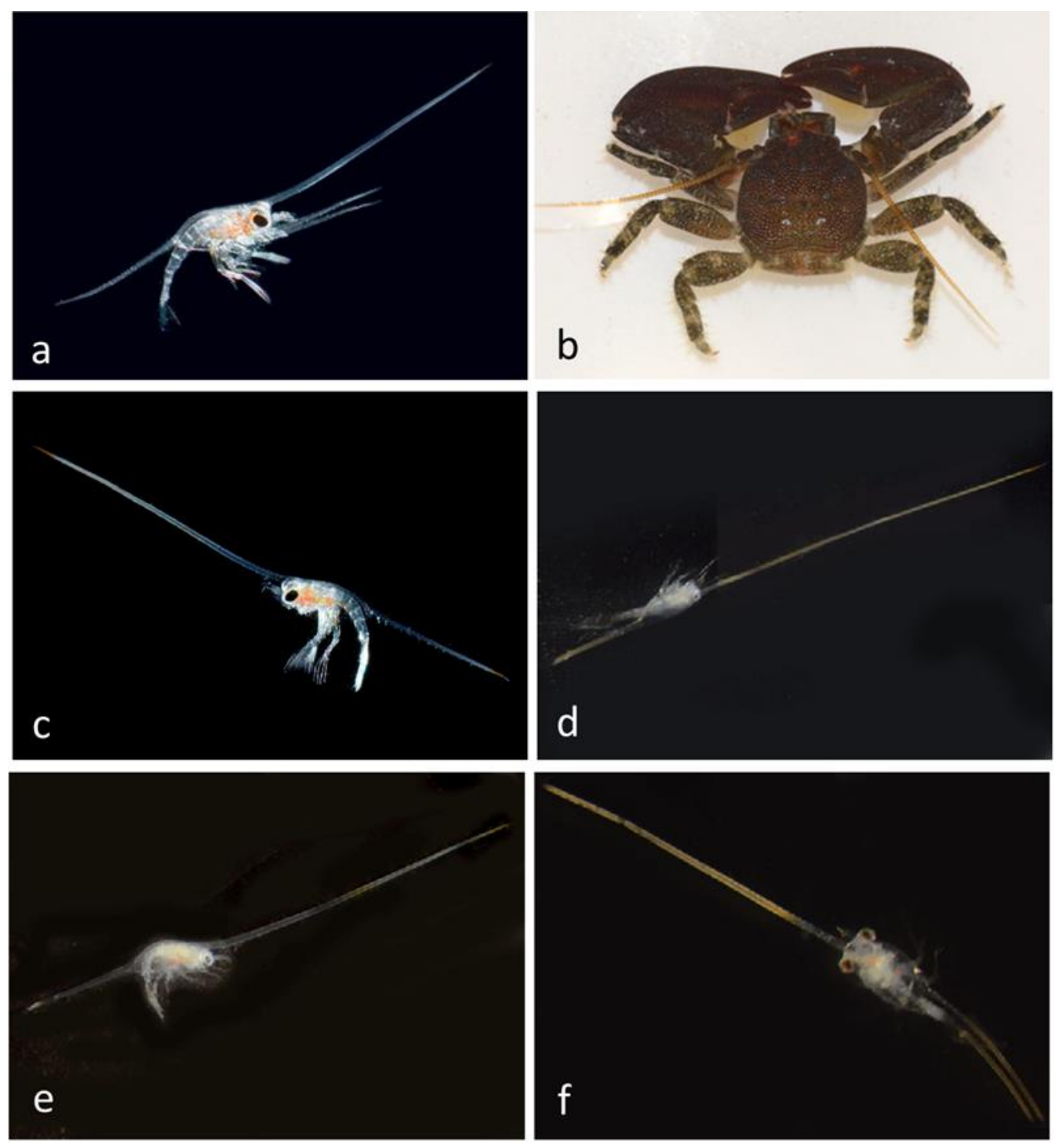

Fig. 32 Order Decapoda: Family Porcellanidae: a,b. Petrolisthes cabrilloi (BIN ACM3389; n=5 zoeae) (Cabrillo's Porcelain Crab). a, Zoea BIOUG01212-A01 from Ocean off Newport Beach. b, Adult (No BIN) BIOUG19282-B07 from Newport Pier. c-f, Family Porcellanidae, zoea larvae. c, BIOUG01207-A06 (BIN ACK8976; n=1) off Newport Beach, CA. d, CCDB 24625 F03 (BIN ADM9208; n=1) from Baja California. e, CCDB 24625 F02 (BIN ADM9207; n=1) from Baja California. f, CCDB 24625 F04 (BIN ADM9098; n=1) from Baja California.

Family Portunidae: An adult of the Swimming Crab Callinectes arcuatus (Figure 33a) was collected at Bahia de Los Angeles, Baja California, and identified by DNA barcode. A megalops larva of Portunus xantusii [16](Figure 33b) from off Newport Aquatic Center was identified by DNA barcode and is shown with an adult (not sequenced) from Newport Bay (Figure 33c). 


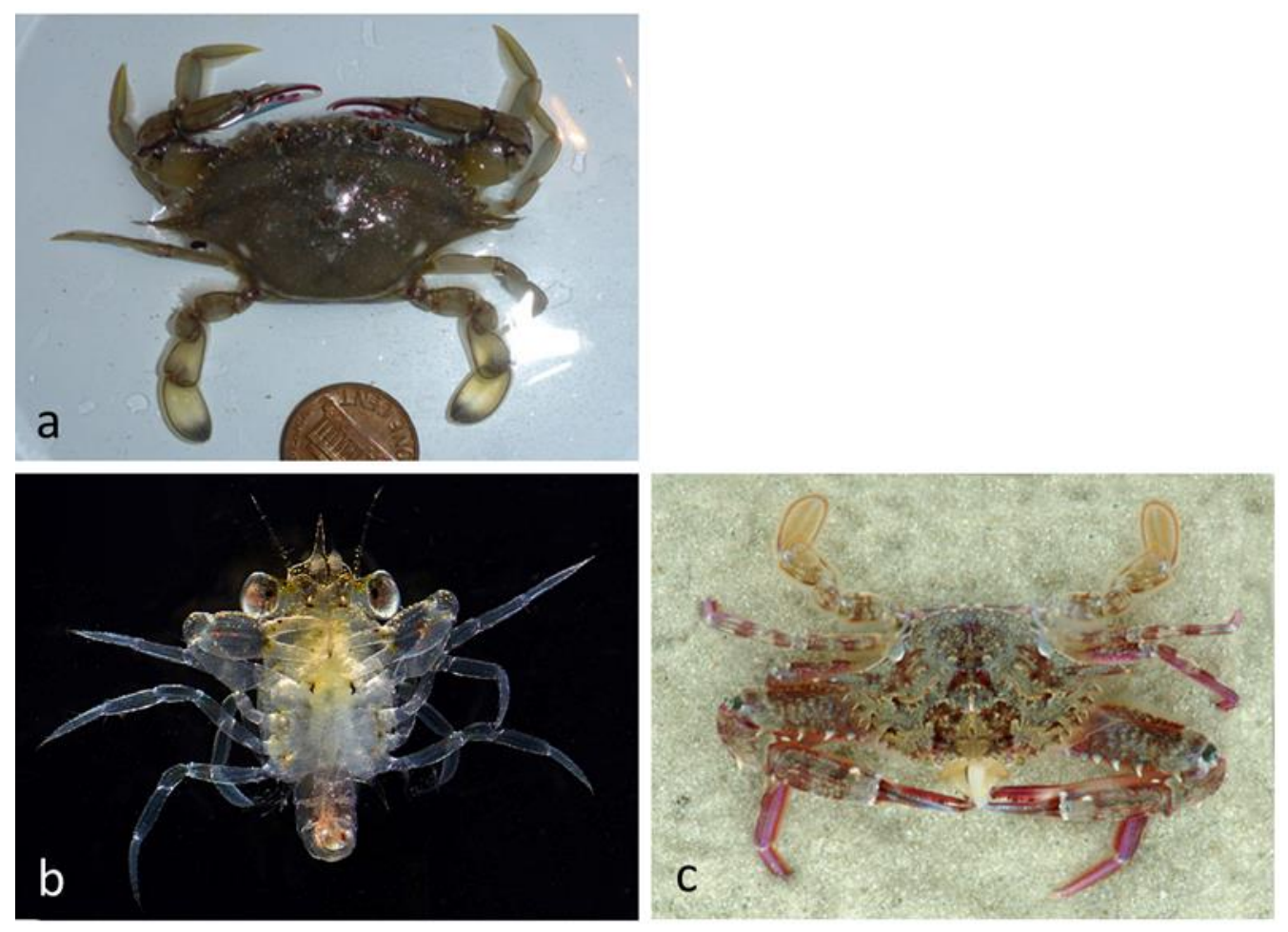

Fig. 33 Order Decapoda: Family Portunidae. a, Callinectes arcuatus (Swimming Crab) (BIN ACD2590; n=3) at Bahia de Los Angeles, Baja California Adult CCDB 24625 H06. b, Portunus xantusii (BIN AAY3148; n=1) Megalops BIOUG01207-D08 from off Newport Aquatic Center. c, Portunus xantusii (No DNA sequence) adult from Upper Newport Bay, Newport Beach, Orange County, CA. 8/16/08.

Family Thoridae: Heptacarpus brevirostris (Broken-back shrimp) [16] one larva and one adult from Balboa at Coral, matched by DNA barcode (Figure 34).
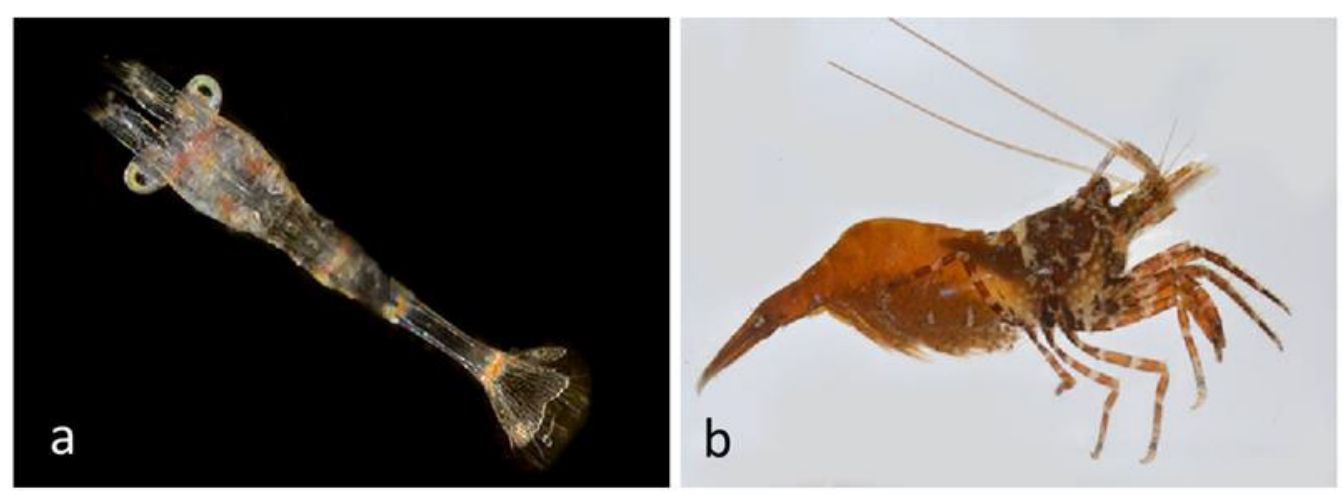

Fig. 34 Order Decapoda: Family Thoridae. Heptacarpus brevirostris (BIN ACU2262; n=1 larva + 1 adult). a, CCDB31777 H01 larva from Balboa at Coral. b, BIOUG01226-D12 adult from Balboa at Coral.

Family Varunidae: Hemigrapsus oregonensis (Yellow Shore Crab) [16]. Zoea and megalops identified by DNA barcode, and adult recognized by morphology (Figure 35). 

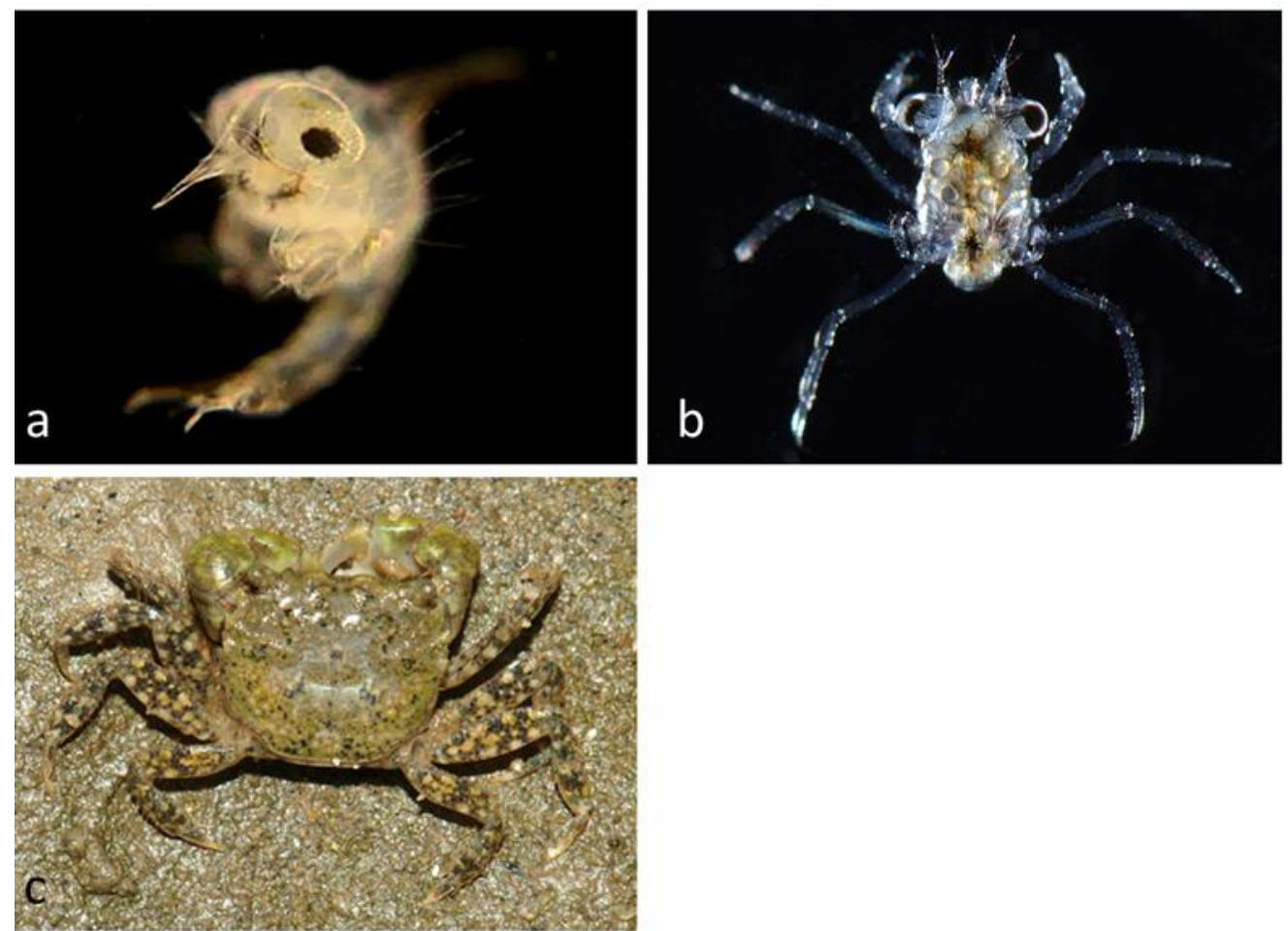

Fig. 35 Order Decapoda: Family Varunidae: Hemigrapsus oregonensis (BIN AAC2203; n=3 zoeae +1 megalops) Yellow Shore Crab. a, Zoea BIOUG19282-A03 off Newport Aquatic Center. b, Megalops BIOUG01207-A11 off Newport Aquatic Center. c, Adult (identified by morphology, not DNA sequence). Shellmaker Island, Upper Newport Bay, Newport Beach, Orange County, CA. 5/24/08.

Family Xanthidae: Paraxanthias taylori (Bumpy Crab) [16] (Figure 36).

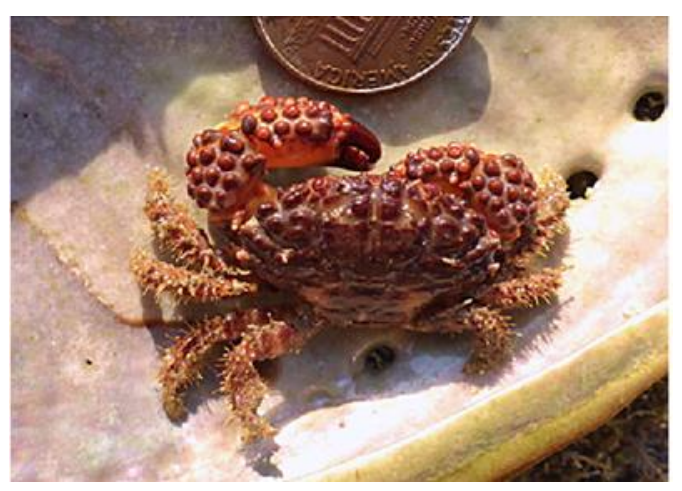

Fig. 36 Order Decapoda: Family Xanthidae: Paraxanthias taylori (Bumpy Crab). (BIN ACH4757; n=1) CCDB 24622 G02 at Laguna Beach, California.

\section{Order Euphausiacea [33]}

Family Euphausiidae: Nyctiphanes simplex adults; Nematoscelis difficilis nauplius larva; Thysanoessa spinifera adult; Euphausia pacifica adults and one nauplius larva were identified by DNA barcode (Figure 37). 

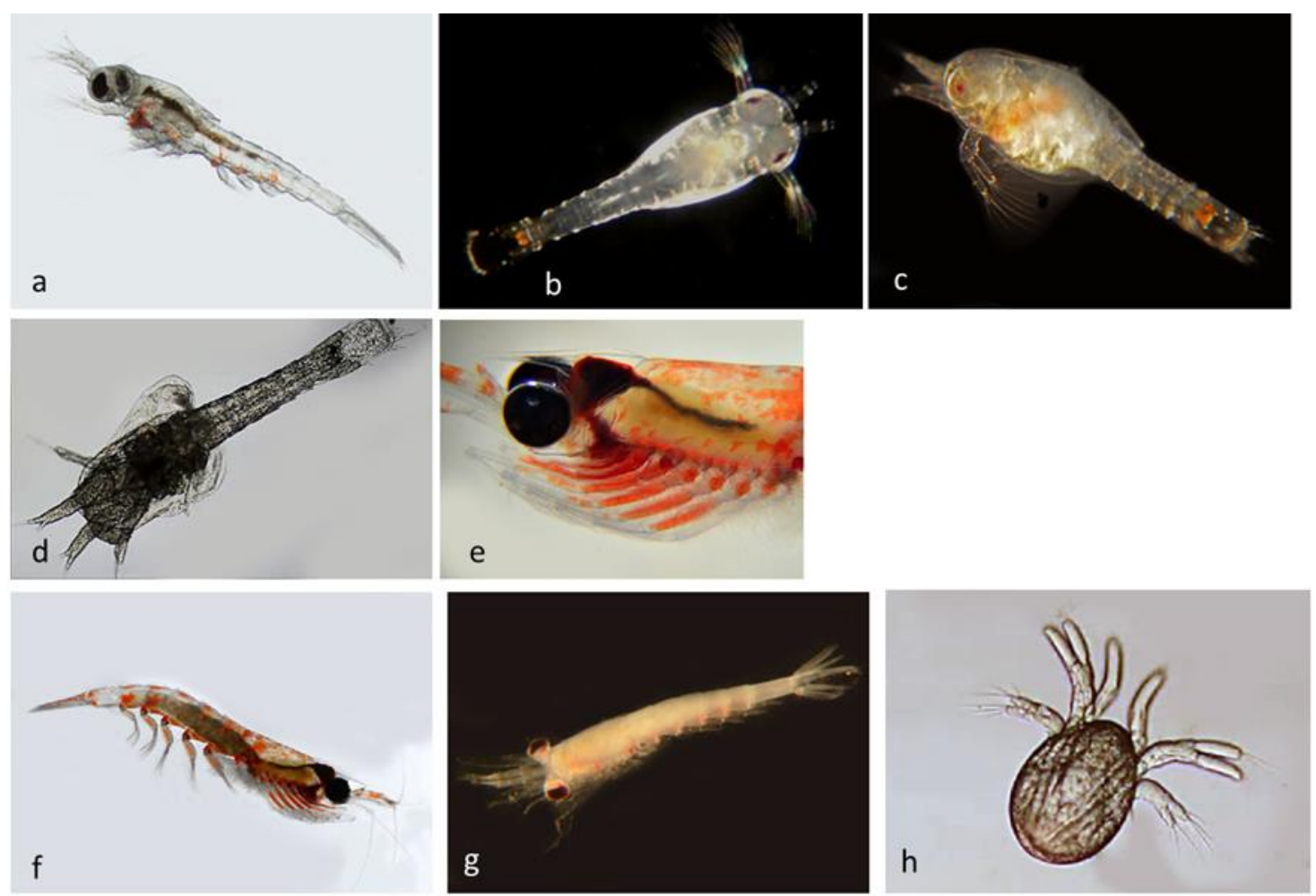

Fig. 37 Order Euphausiacea, Family Euphausiidae. a, Nyctiphanes simplex (BIN AAK4259; n=12) BIOUG19282-F12 from Pacific Ocean off Dana Point. b, Nyctiphanes simplex (BIN AAK4259; n=12) BIOUG01226-B05 from off Dana Point. c, Nyctiphanes simplex (BIN AAK4259 ; n=12) BIOUG19282-G01 from Pacific Ocean off Dana Point. d, Nematoscelis difficilis (BIN AAX6036; n=1) Nauplius larva BIOUG01207-C12 from off Crystal Cove State Park. e, Thysanoessa spinifera (BIN AI0441; n=1) BIOUG19282-F09 from Pacific Ocean off Dana Point. f,g,h: Euphausia pacifica (BIN AAJ0072; $\mathrm{n}=1$ nauplius +2 adults). f, Adult BIOUG19282-F10 from Pacific Ocean off Dana Point. g, Adult BIOUG19282-C08 from off Dana Point. h, Nauplius larva BIOUG01218 H02 from Delhi Channel, Upper Newport Bay.

\section{Order Isopoda [34]}

Family Paranthuridae: Paranthura sp.; Paranthura elegans; Ianiropsis epilittoralis [16]; an unidentified Isopod parasitic on Ghost Shrimp, Neotrypaea californiensis (All adults; Figure 38) identified by DNA barcode.

Family Cymothoidae: Nerocila sp. (Figure 38). 

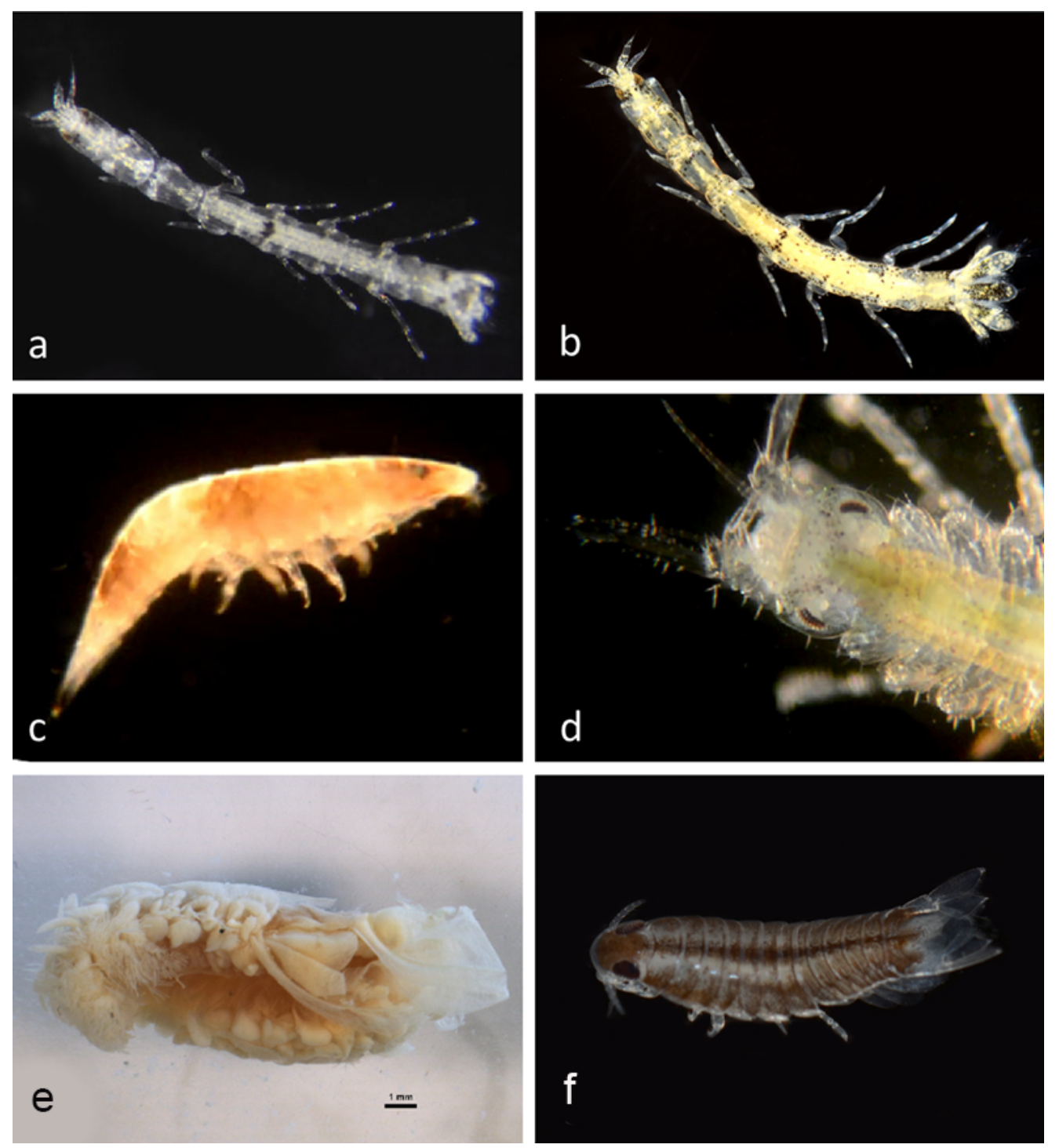

Fig. 38 Class Malacostraca, Order Isopoda. a, Paranthura sp. (BIN ADQ3122; n=1) Family Paranthuridae CCDB 24622 D06 from Newport Beach Harbor entrance. b, Paranthura elegans (no Bar Code) Family Paranthuridae BIOUG01212-D11 off Balboa at Collins. c, Isopod (BIN ADM9094; n=1) CCDB 24625 E01 from Bahia de Los Angeles. d, Ianiropsis epilittoralis (BIN AAE6126) CCDB-32335 H05 from eelgrass at Balboa Pavilion. e, Isopod (BIN ACL8004; n=2) BIOUG01212-C12 from near Balboa Pavilion. Parasitic on Ghost Shrimp, Neotrypaea californiensis. f, Nerocila sp. (BIN ACK9256; n=1) Family Cymothoidae BIOUG01207-B09 from Bayview Way/Jamboree Upper Newport Bay.

\section{Order Mysida}

Family Mysidae: Six unidentified species were distinguished by DNA barcode (Figure 39). Two specimens, morphologically mysids, gave DNA barcodes corresponding to the Sheep Crab Loxorhynchus grandis [16](Figure 17e,f). It seems likely that this is due to their having consumed Loxorhynchus grandis larvae and that the PCR amplification amplified the Loxorhynchus grandis DNA barcode rather than the mysid sequence. 

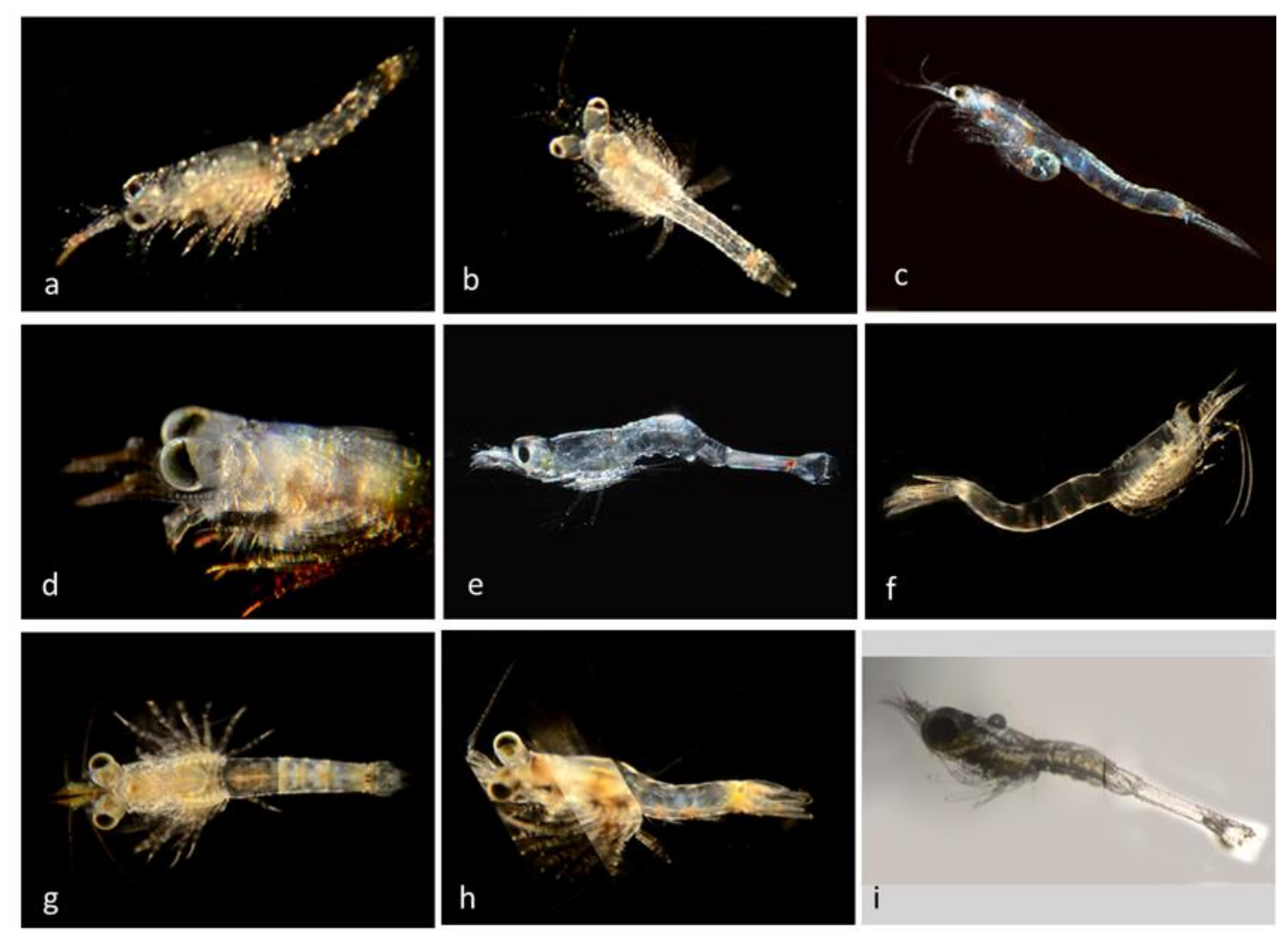

Fig. 39 Order Mysida, Family Mysidae. a, Mysid (BIN ACK9354; n=3) BIOUG01218- C04 off Newport Pier. b, Mysid (BIN ACK9354; n=3) BIOUG01207-G04 off Newport Pier. c, Mysid (BIN ACK9354; n=3) BIOUG01212-A10 off Newport Pier. Female showing brood pouch. d, Mysid (BIN ACK9353; n=1) CCDB-32335 F12 off Balboa at Coral. e, Mysid (BIN ACK9237; n=2) BIOUG01207-A02 from Pacific Ocean off Newport Beach. f, Mysid (BIN ACN0139; n=3) BIOUG01226G08 off Lido Island at Genoa. g, Mysid (BIN ACU2262; n=2) BIOUG01226-G11 off Newport Pier. h, Mysid (BIN ACU2262; n=2) BIOUG01226-H06 off Newport Pier. i, Mysid (BIN ACK9540; n=1) BIOUG01207-H09 from Pacific Ocean off Crystal Cove.

Order Tanaidacea [35]. Tanaids do not undergo a true planktonic stage. The early developmental period is spent while young are within the marsupium of the mother. Subsequently, post-larvae emerge as epibenthic forms.

Two individuals of a species of Zeuxo sp., and three unidentified Tanaids were identified by DNA barcode (Figure 40). 

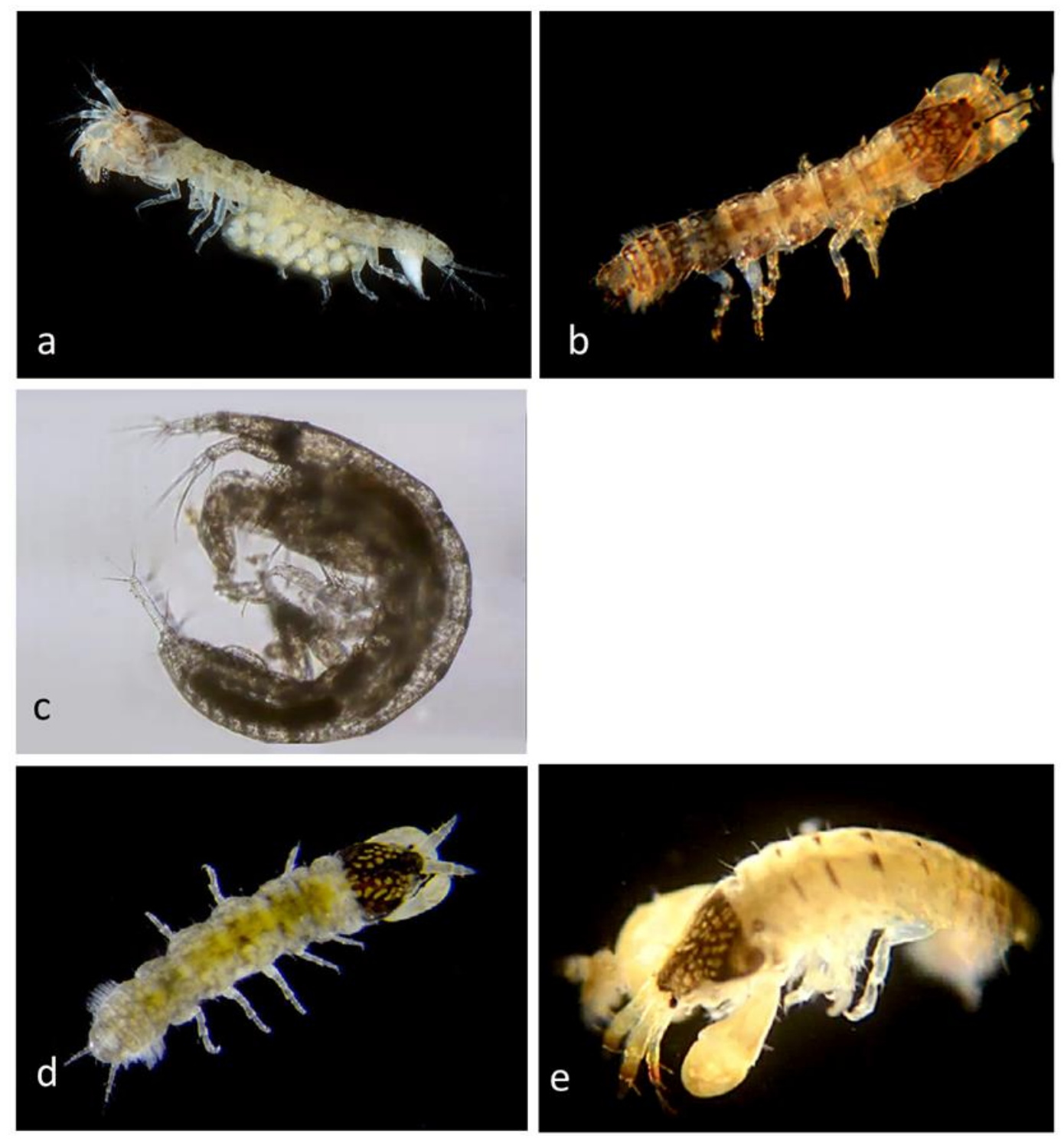

Fig. 40 Class Malacostraca, Order Tanaidacea. a, Zeuxo sp. (BIN ACU2492; n=2) BIOUG01212-D12. Off Balboa Island at Collins. b, Zeuxo sp. (BIN ACU2492; n=2) CCDB-32335 H04. On eelgrass at Balboa Pavilion. c, Unknown sp. (BIN ACM3687; n=1) BIOUG01212-E09 off Balboa at Coral. d, Tanaidae (No BIN) CCDB-24003 H11 off Back Bay Science Center. e, Tanaidae (BIN ACN0972; n=1) BIOUG01218 A08 off Lido Island.

\section{Discussion}

DNA barcoding has often revealed unexpected species diversity in many taxa $[17,36,37]$ and the present study leads to the same conclusion for marine crustacea. It shows the validity of this approach and the utility of the COI Barcode for identifying species. Furthermore, our data show a clear "DNA barcode gap"; i.e. a much larger range of interspecific divergences versus intraspecific divergences in this DNA sequence for crustacea (Fig 41). 


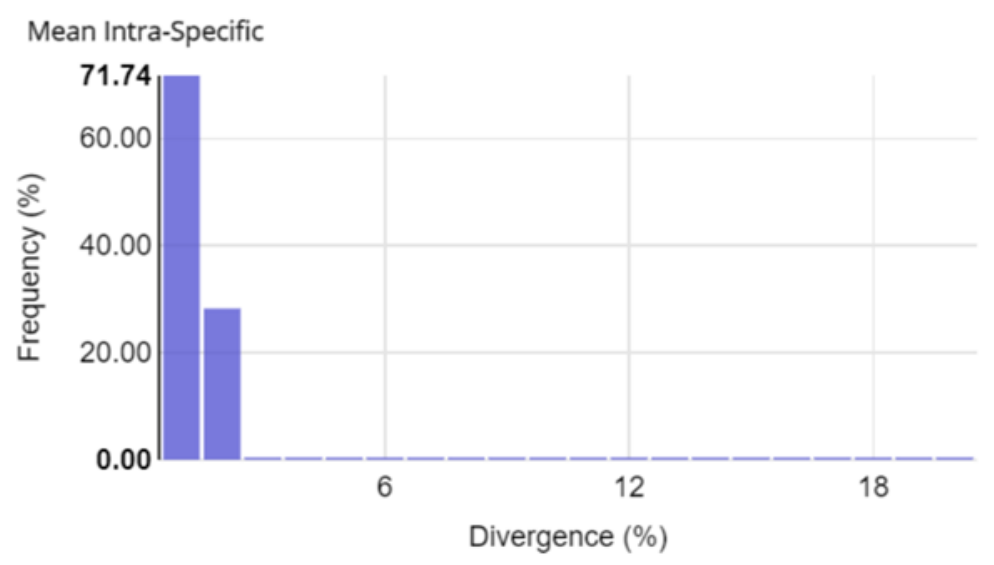

Distribution Details

Min: 0 Mean: 0.37

Max: 0 SE: 0.01

Distance to Nearest Neighbour

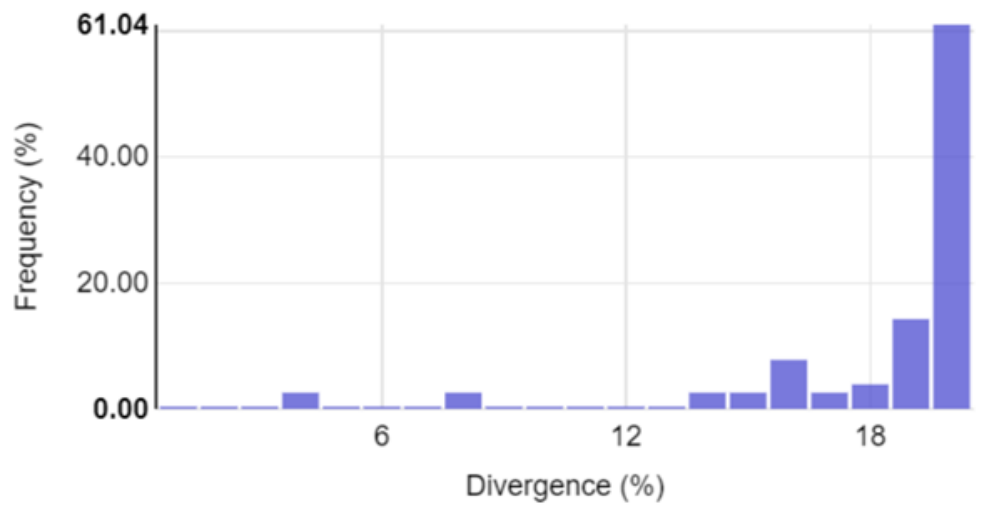

Distribution Details

Min: 3.36 Mean: 21.17

Max: 56.46 SE: 0.1

Fig. 41 Sequence divergence in the COI Barcode for intraspecific and interspecific comparisons using the data included in this publication. Distance Model: Kimura 2 Parameter; Alignment: BOLD Aligner; Length filter $>/=300 \mathrm{bp}$; Excluded: contaminants and misidentifications, records with stop codons

The DNA differences in the COI barcode are probably not responsible for the morphological differences we have observed between specimens in separate taxa. However, the DNA barcode differences that have evolved between morphologically distinct organisms can be used to examine the degree of relatedness between them. When the DNA sequence data are organized into a taxonomic tree, the results are generally consistent with the taxonomic tree according to conventional morphological methods. This can be examined most effectively by cladistic analysis, which in this context involves examination of the taxonomic tree for "DNA clades" - groups of species that are uniquely and exclusively related by DNA sequence. According to this analysis, all the calanoid copepods constitute a DNA clade, although the cyclopoid and harpacticoid copepods are mixed. The Decapods apart from the Palaemonidae constitute a DNA clade while the one example of Palaemonidae appears to be more distantly related. The Ampeliscidae and some relatives constitute one clade of Amphipods, while the remainder of the Amphipods constitute another larger clade which includes two separate clades of Caprellids within it. The Tanaids; all the barnacles; the Cladocerans 
including Penilia; the Euphausiids; the Isopods and the Ostracods present as distinct clades, while the Mysids group as several clades mixed with the Cumaceans and Decapods.

One of the major goals of this study was to use DNA barcoding to match life-cycle stages within individual species, and this has been successful. DNA barcoding was used to identify all three major stages (zoea, megalops, adult) of the Lined Shore Crab, Pachygrapsus crassipes, which is one of the commonest intertidal decapods in our area. Zoea and megalops of the Yellow Shore Crab Hemigrapsus oregonensis were identified and the adult has been collected earlier at the Back Bay Science Center in Newport Bay.

In other cases, larval stages were identified by DNA barcoding, which suggests the presence of adult populations, but the adults have not yet been found, possibly because they are in subtidal areas where not enough sampling has been done. For example, the zoea and megalops of the yellow rock crab Metacarcinus anthonyi and a megalops of the graceful rock crab M. gracilis, were identified, but no adults of these species were found. The finding of many larval specimens in different stages suggest the existence of nearby breeding adult populations. In the family

Pinnotheridae (Pea crabs), 19 zoeae but no adults of Pinnixa franciscana, and one zoea of Pinnixa tubicola were identified. Adults of Pea crabs live in burrows of Callianassa (mud shrimp) and Urechis (Innkeeper worm), where we have not collected. Our collections include zoea of the Spiny Mole Crab Blepharipoda occidentalis, which is difficult to find but is known to be a common local resident.

The larval stages of meroplanktonic species can take advantage of ocean currents, drifting for dispersal and range expansion [38]. In addition to swimming, organisms can attach to floating objects to feed, rest, and travel great distances. For some, including crabs of the genus Planes, rafting is a way of life. Larvae of many species can delay settlement and metamorphosis if an appropriate settlement site is not found, and in some cases without a settlement site the larvae will die, fail to molt to the next stage, or be consumed as prey [39]. Larvae of the barnacle Chthamalus dalli are found in many Newport Bay samples and in the immediate areas north and south of the harbor entrance. No adults have been found but it seems likely they will be found on boat bottoms rather than rocks.

Amphibalanus eburneus, a Western Atlantic barnacle, was first found in the Pacific Ocean in Colorado Lagoon, Long Beach, California in 2000 [30]. Colorado Lagoon is 10 miles NW of Newport Bay and 2 miles SE of the Port of Los Angeles. Nauplii were identified from two locations in Newport Bay, suggesting a range extension from Colorado Lagoon since 2000, consistent with the statement "Based on its broad tolerances, this species has the potential to expand its range on the Pacific coast" [30]. These larval catches suggest that adults will be found in our sampling areas.

Two adult sequences of Amphibalanus amphitrite were obtained from the Salton Sea, an inland, brackish-water body, in Southern California, north of the upper reaches of the Gulf of California, without ocean contact either to the Pacific or to the Gulf of California. One nauplius larva of this species was found in Newport Bay.

Sometimes, a specimen of an exotic species may have arrived in ballast water of ships, rather than representing a continuing presence in the ecosystem. For example, one cypris larva of Megabalanus rosa, a Western Pacific barnacle species, was found in the entrance to Newport Harbor. In Newport Bay one larva of the long-wristed hermit crab, Pagurus longicarpus, was identified; this species is common along the Atlantic and Gulf coasts of the United States and the Atlantic coast of Canada [40]. Since there are apparently no other known records of these species in our area, they are unlikely to be from a local population and are more likely to have arrived from discharge of ballast water [41] possibly from the nearby port of Long Beach. However, our finding of a zoea suggests a breeding population somewhere in Orange County between Newport Bay and the Port of Los Angeles. These larvae make it important to search for adults as other benthic species are sampled. Water sampling for planktonic larvae is much simpler than searching for adults, and DNA barcoding can be more definitive than morphological identification, especially for early larval stages. 


\section{References}

1. Watling L, Thiel M. Functional Morphology and Diversity: Volume I (The Natural History of the Crustacea, 1). Oxford University Press, Oxford.; 2012. https://books.google.com/books?hl=en\&lr=\&id=HJ5pAgAAQBAJ\&pgis=1. 2. Perry R. A Guide to the Marine Plankton of southern California. 3rd. Los Angeles, CA: UCLA OceanGLOBE \& Malibu High School; 2003. http://www.cosee.net/cosee-west/Jun23-272008/guide_plankton1.pdf.

3. Perry RB. Ocean Images: the Plankton: Photographic Guide to Identifying Common Plankton. 2nd edition. Blurb; 2008. https://www.blurb.com/b/277427-ocean-images-the-plankton-2nd-edition.

4. Perry RB. Ocean Images: the Plankton, with emphasis on plankton from Southern California. 2nd. Blurb Books; 2008. https://www.blurb.com/user/zalophus.

5. Williams TA. The Nauplius Larva of Crustaceans: Functional Diversity and the Phylotypic Stage1. American Zoologist. 1994;34:562-9. doi:10.1093/icb/34.4.562.

6. Lee WL, Miller MA. Isopoda and Tanaidacea: The Isopods and Allies. In: Morris RH, Abbott DP, Haderlie EC, editors. Intertidal Invertebrates of California. Original. Stanford, CA: Stanford University Press; 1980. p. 536-58.

7. Olesen J. Monophyly and Phylogeny of Branchiopoda, with Focus on Morphology and Homologies of Branchiopod Phyllopodous Limbs. Journal of Crustacean Biology. 2007;27:165-83. doi:10.1651/S-2727.1.

8. Richter, Scholtz. Phylogenetic analysis of the Malacostraca (Crustacea). Journal of Zoological Systematics and Evolutionary Research. 2001;39:113-36. doi:https://doi.org/10.1046/j.1439-0469.2001.00164.x.

9. de Grave S, Pentcheff ND, Ahyong ST, Chan T-Y, Crandall KA, Dworschak PC, et al. A Classification of Living and Fossil Genera of Decapod Crustaceans. Raffles Bulletin of Zoology. 2009;Supplement 21:1-109.

10. Essock-Burns T, Gohad N v, Orihuela B, Mount AS, Spillmann CM, Wahl KJ, et al. Barnacle biology before, during and after settlement and metamorphosis: a study of the interface. The Journal of Experimental Biology. 2017;220:194207. doi:10.1242/jeb.145094.

11. Reaka-Kudla ML. Crustaceans. In: Encyclopedia of Biodiversity: Second Edition. Elsevier Inc.; 2001. p. 396-418.

12. Zhang Z-Q. Animal biodiversity: an outline of higher-level classification and survey of taxonomic richness -

Phylum Arthropoda von Siebold, 1848. Zootaxa. 2011;4138:99-103.

13. Turner J. The importance of small planktonic copepods and their roles in pelagic marine food webs. Zoological Studies. 2004;43:255-66.

14. Light SF, Carlton JT. The Light and Smith Manual: Intertidal Invertebrates from Central California to Oregon. 4th edition. University of California Press; 2007. https://books.google.com/books?id=64jgZ1CfmB8C.

15. Hebert PDN, Cywinska A, Ball SL, DeWaard JR. Biological identifications through DNA barcodes. Proceedings of the Royal Society B: Biological Sciences. 2003;270:313-21.

16. Barwick K, Cadien DB, Lovell LL, editors. A Taxonomic Listing of Benthic Macro-and Megainvertebrates. 12th edition. Los Angeles, CA: The Southern California Association of Marine Invertebrate Taxonomists; 2018.

17. Bryant PJ, Arehart TE. Diversity and life-cycle analysis of Pacific Ocean zooplankton by videomicroscopy and DNA barcoding: Hydrozoa. PLoS ONE. 2019;14:1-22.

18. Raupach MJ, Barco A, Steinke D, Beermann J, Laakmann S, Mohrbeck I, et al. The application of DNA barcodes for the identification of marine crustaceans from the North Sea and adjacent regions. PLoS ONE. 2015;10.

19. Bezeng BS, van der Bank HF. DNA barcoding of southern African crustaceans reveals a mix of invasive species and potential cryptic diversity. PLoS ONE. 2019;14:1-12. doi:10.1371/journal.pone.0222047.

20. Radulovici AE, Sainte-Marie B, Dufresne F. DNA barcoding of marine crustaceans from the Estuary and Gulf of St Lawrence: A regional-scale approach. Molecular Ecology Resources. 2009;9 SUPPL. 1:181-7.

21. Raupach MJ, Radulovici AE. Looking back on a decade of barcoding crustaceans. ZooKeys. 2015;539:53-81. 
22. Zaiko A, Samuiloviene A, Ardura A, Garcia-Vazquez E. Metabarcoding approach for nonindigenous species surveillance in marine coastal waters. Marine Pollution Bulletin. 2015;100:53-9. doi:https://doi.org/10.1016/j.marpolbul.2015.09.030.

23. Martin J, Davis G. An updated classification of the recent crustacea. Science Se. 2001.

24. Mauchline J, Blaxter JHS, Southward AJ, Tyler PA. The Biology of Calanoid Copepods. Advances in Marine Biology. 1998;:1-660.

25. Khodami J.V. McArthur, L. Blanco-Bercial \& P. Martínez Arbizu S. Molecular phylogeny and revision of copepod Orders (Crustacea: Copepoda). Scientific Reports. 2017;7:1-11. doi:10.1038/s41598-017-06656-4.

26. Trujillo-ortiz A. Life cycle of the marine calanoid copepod Acartia californiensis Trinast reared under laboratory conditions. 1986;XXVII January 1986:189-204.

27. Pérez-Losada M, Høeg JT, Simon-Blecher N, Achituv Y, Jones D, Crandall KA. Molecular phylogeny, systematics and morphological evolution of the acorn barnacles (Thoracica: Sessilia: Balanomorpha). Molecular Phylogenetics and Evolution. 2014;81:147-58. doi:https://doi.org/10.1016/j.ympev.2014.09.013.

28. Anger K. Contributions of larval biology to crustacean research: a review. Invertebrate Reproduction \& Development. 2006;49:175-205. doi:10.1080/07924259.2006.9652207.

29. Carlton JT, Newman WA, Pitombo FB. Barnacle Invasions: Introduced, Cryptogenic, and Range Expanding Cirripedia of North and South America. In: Galil BS, Clark PF CJ, editor. In the Wrong Place - Alien Marine Crustaceans: Distribution, Biology and Impacts. 2011. p. 159-213.

30. Fofonoff PW, Ruiz GM, Steves B, Simkanin C, Carlton JT. National Exotic Marine and Estuarine Species Information System. 2020. invasions.si.edu/nemesis/citation.html. Accessed 25 Oct 2020.

31. Brandão SN, Angel SN, Karanovic I, Perrier V, Meidla T. World Ostracoda Database. Last access: 01 July 2020. 2020. http://www.marinespecies.org/ostracoda.

32. Watling, L.; Gerken S. World Cumacea Database. 2021. doi:10.14284/354.

33. Mauchline J. The Biology of Mysids and Euphausiids. Advances in Marine Biology. 1980;18:3-681.

34. Brusca RC, Coelho V, Taiti S. A guide to the coastal isopods of California. Tree of Life Web Project. 2001;:http://works.bepress.com/vania-coelho/4/. http://tolweb.org/notes/?note_id=3004.

35. Blazewicz-Paszkowycz M, Bamber R, Anderson G. Diversity of Tanaidacea (Crustacea: Peracarida) in the World's Oceans - How Far Have We Come? PLoS ONE. 2012;7:1-11. https://doi.org/10.1371/journal.pone.0033068.

36. Bucklin A, Steinke D, Blanco-Bercial L. DNA Barcoding of Marine Metazoa. Annual Review of Marine Science. 2011;3:471-508.

37. Waugh J. DNA barcoding in animal species: Progress, potential and pitfalls. BioEssays : news and reviews in molecular, cellular and developmental biology. 2007;29:188-97.

38. Strathmann R, Hughes T, Kuris A, Lindeman K, Morgan S, Pandolfi J, et al. Evolution of self-recruitment and its consequences for marine populations. Bull Mar Sci. 2002;70:377-96.

39. Pechenik J. Delayed metamorphosis by larvae of benthic marine-invertebrates - does it occur? Is There a Price to Pay? Ophelia. 1990;32:63-94.

40. ITIS. Integrated Taxonomic Information System. http://www.itis.gov. Accessed 31 May 2021.

41. Elçiçek H, Parlak A, Cakmakcı M. Effect of Ballast Water on Marine and Coastal Ecology. Journal of Selcuk University Natural and Applied Science. 2013;1:454-63.

42. Hebert PDN, Cywinska A, Ball SL, DeWaard JR. Biological identifications through DNA barcodes. Proceedings of the Royal Society B: Biological Sciences. 2003;270:313-21.

43. Folmer O, Black M, Hoeh W, Lutz R, Vrijenhoek R. DNA primers for amplification of mitochondrial cytochrome c oxidase subunit I from diverse metazoan invertebrates. Molecular marine biology and biotechnology. 1994;3:294-9. 